\title{
Transcriptome landscape of the human placenta
}

\author{
Jinsil Kim ${ }^{1+}$, Keyan Zhao ${ }^{2+}$, Peng Jiang ${ }^{2 \dagger}$, Zhi-xiang Lu' ${ }^{2}$, Jinkai Wang ${ }^{2}$, Jeffrey C Murray ${ }^{3,4,5^{*}}$ and Yi Xing ${ }^{2,6,7^{*}}$
}

\begin{abstract}
Background: The placenta is a key component in understanding the physiological processes involved in pregnancy. Characterizing genes critical for placental function can serve as a basis for identifying mechanisms underlying both normal and pathologic pregnancies. Detailing the placental tissue transcriptome could provide a valuable resource for genomic studies related to placental disease.

Results: We have conducted a deep RNA sequencing (RNA-Seq) study on three tissue components (amnion, chorion, and decidua) of 5 human placentas from normal term pregnancies. We compared the placental RNA-Seq data to that of 16 other human tissues and observed a wide spectrum of transcriptome differences both between placenta and other human tissues and between distinct compartments of the placenta. Exon-level analysis of the RNA-Seq data revealed a large number of exons with differential splicing activities between placenta and other tissues, and $79 \%$ (27 out of 34) of the events selected for RT-PCR test were validated. The master splicing regulator ESRP1 is expressed at a proportionately higher level in amnion compared to all other analyzed human tissues, and there is a significant enrichment of ESRP1-regulated exons with tissue-specific splicing activities in amnion. This suggests an important role of alternative splicing in regulating gene function and activity in specific placental compartments. Importantly, genes with differential expression or splicing in the placenta are significantly enriched for genes implicated in placental abnormalities and preterm birth. In addition, we identified 604-1007 novel transcripts and 494-585 novel exons expressed in each of the three placental compartments.
\end{abstract}

Conclusions: Our data demonstrate unique aspects of gene expression and splicing in placental tissues that provide a basis for disease investigation related to disruption of these mechanisms. These data are publicly available providing the community with a rich resource for placental physiology and disease-related studies.

Keywords: Placenta, Amnion, Chorion, Decidua, RNA-Seq, Transcriptome, Alternative splicing, Functional interaction network, Novel transcriptional active region

\section{Background}

Pregnancy and parturition require an intricate interplay between maternal and fetal factors, orchestrated by the placenta, which lies at the interface between mother and fetus. The placenta performs multiple functions critical for fetal survival, growth, and development, including transport of gases, nutrients, and waste products, hormone production, protection of the fetus from maternal immune attack, and anchorage of the fetus to the uterus [1]. The role of the placenta as a key organ of pregnancy is well demonstrated by the fact that placental pathology

\footnotetext{
* Correspondence: jeff-murray@uiowa.edu; yi-xing@uiowa.edu

† Contributed equally

${ }^{2}$ Department of Internal Medicine, University of lowa, lowa City, IA 52242, USA

${ }^{3}$ Department of Pediatrics, University of lowa, lowa City, IA 52242, USA Full list of author information is available at the end of the article
}

is associated with adverse maternal and fetal outcomes such as preterm birth (PTB), intrauterine growth restriction (IUGR), and preeclampsia (PE) [1-3].

The value of placental examination is well recognized in the setting of PTB, for instance, which complicates over $12 \%$ of all pregnancies in the U.S. [3-5]. Histological examination of the placenta, which is frequently carried out to explore possible causes of preterm delivery, has been a useful tool for identifying lesions commonly associated with PTB, such as chorioamnionitis [3]. In cases where no remarkable histologic abnormalities are found, investigation into molecular alterations causing placental dysfunction could provide insight into the pathogenesis of prematurity.

The normal function of the placenta depends on its structural integrity, and the proper growth and development of its structural components require the finely
C Biomed Central

(c) 2012 Kim et al; licensee BioMed Central Ltd. This is an Open Access article distributed under the terms of the Creative Commons Attribution License (http://creativecommons.org/licenses/by/2.0), which permits unrestricted use, distribution, and reproduction in any medium, provided the original work is properly cited. 
tuned regulation of relevant genes. Thus, alterations in gene expression and RNA processing may represent one of the major molecular mechanisms underlying pathological pregnancies. Previously, numerous studies have investigated changes in global human placental gene expression associated with gestational age [6], physiologic labor $[7,8]$ or pathological conditions [9]. The two most comprehensive gene expression profiling studies related to the placenta used microarray analysis to characterize four different components of the human placenta in 76 individuals [10] and the mouse placenta over the whole course of pregnancy [11]. Although those microarray studies have provided useful insights into the placental transcriptome, they were limited in depth in that they only examined gene-level expression changes, and did not have the resolution to investigate the complexity of the placental transcriptome that arises from changes in RNA processing.

Alternative splicing (AS) is a common mechanism of gene regulation in higher eukaryotes, occurring in over $90 \%$ of multi-exon genes in the human genome $[12,13]$. AS is regulated by complex interactions between cis-acting splicing elements and trans-acting factors [14]. Many splicing regulators have tissue-specific expression patterns, resulting in widespread differences in AS patterns across different tissues. In addition to playing a critical role in regulating normal gene functions, AS is also frequently involved in diseases $[15,16]$. Previous studies have revealed associations between AS of individual genes and human pregnancy complications [17-19]. For example, the soluble isoform of the fms-like tyrosine kinase-1 (sFlt1) arising from AS and polyadenylation is significantly up-regulated in placentas of women with PE [19], and encodes a potent inhibitor of the vascular endothelial growth factor (VEGF) [18]. Despite such interesting anecdotal examples, the global patterns of AS of human genes have not been examined systematically in the placenta.

In this study, we used high-throughput RNA-Seq to conduct a genome-wide analysis of the normal placental transcriptome. RNA-Seq is a powerful technology for transcriptome analysis that allows global characterization of gene expression and AS at the nucleotide resolution [20]. Given the heterogeneity in tissue composition of the placenta and the importance of both fetal and maternal factors in normal and pathological pregnancy, we separately examined three placental tissue components: the amnion and chorion of fetal origin, and the maternally derived decidua [1]. The amnion and chorion were obtained from the extraplacental membranes (reflected membranes), which provide a purer source of the fetal membranes compared with those overlying the chorionic plate. The decidua was dissected from the surface of the basal plate of the placenta, which has close relevance to normal placental physiology. We observed a wide spectrum of gene-level and exon-level transcriptome differences both between placenta and other human tissues and between distinct compartments of the placenta. Our work provides the first high-resolution profiles of gene expression and AS characteristic of different parts of the normal human placenta.

\section{Results}

\section{Overview of the RNA-Seq data}

We sequenced pooled mRNA of amnion, chorion, and decidua separately taken from five normal term placentas ( 3 from male infants and 2 from female infants). For each of the placental tissues, we generated 2 lanes of paired-end Illumina RNA-Seq data with 54 bp and 72 bp in read length and 23-33 million reads of each lane, for a total of 50-60 million paired-end reads per tissue. We only used $50 \mathrm{bp}$ of each end for mapping and analysis based on the sequencing error profile. In addition, we also obtained the Illumina Human Body Map 2.0 (HBM2.0) data with 73-83 million 50 bp paired-end reads from 16 normal non-placental human tissues (adipose, adrenal, brain, breast, colon, heart, kidney, liver, lung, lymph node, ovary, prostate, skeletal muscle, testes, thyroid and white blood cells). We mapped the sequence reads of each tissue to the reference human genome sequence (hg19) as well as all possible exonexon junctions (Ensembl genes, r57). We obtained a high mapping rate with $70-90 \%$ and $7-10 \%$ of reads mapped to the reference genome and exon-exon junctions, respectively (Table S1 in Additional file 1). 70$80 \%$ of the mapped paired-end reads were uniquely mapped pairs and were used for subsequent analysis.

\section{Global analysis of gene expression in placenta and other human tissues}

Using the uniquely mapped read pairs, we estimated the expression levels of 22,523 protein-coding genes (Ensembl genes, r57) in each tissue using the "Fragments Per Kilobase of gene per Million mapped fragments" (FPKM) metric [21] in a way similar to RPKM [22] (see details in Methods). With a coverage depth ranging from 50 to 80 million paired-end reads per tissue, we detected the expression (i.e. FPKM > 0) of the majority of the protein-coding genes $(66-84 \%$ for each of the 19 tissues). Approximately half of the genes were expressed with FPKM > 1 (Table S2 and Figure S1 in Additional file 1). We investigated the similarity in the global gene expression profiles among the three placental compartments and 16 HBM2.0 tissues using average linkage hierarchical clustering of the top 1,000 most divergent genes (Figure 1). The three placental tissues clustered more closely with one another than with the other 16 tissues, suggesting the existence of a placenta- 


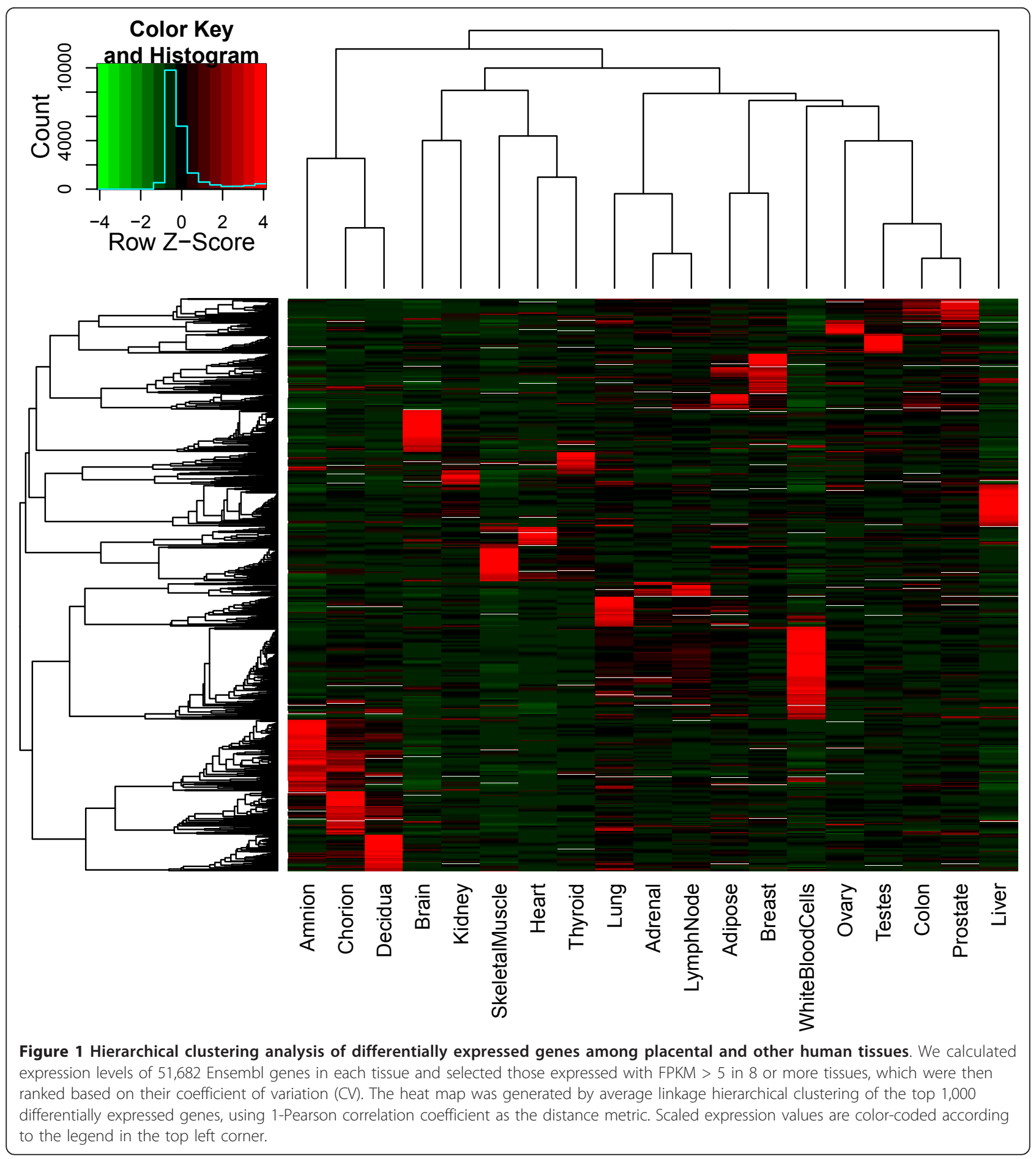

specific gene expression signature. In addition, we also observed genes with distinct expression patterns among amnion, chorion, and decidua, indicating that each compartment of the placenta has its unique expression signature, possibly reflecting differences in their functions and/or biological activities.
To obtain a more detailed picture of genes potentially important for normal placental function, we compared the RNA-Seq gene expression profiles between the three placental compartments and the 16 HBM2.0 tissues to identify two types of genes with preferential expression in the placenta: (1) placenta-enriched genes, defined as 
genes with an FPKM value of at least 1 and greater than 4-fold difference in FPKM between any of the three placental tissues and the average of the 16 non-placental tissues as similarly defined in another study [11]; and (2) placenta-specific genes, defined as genes whose RNASeq reads were only detected in the placenta but not in any of the 16 non-placental tissues.

We identified 938, 865, and 944 genes with at least 4fold enriched expression in amnion, chorion, and decidua, respectively, as compared to non-placental tissues, including 216 genes shared among the three compartments of the placenta. We also used a similar strategy to generate a list of 758 placenta-enriched genes using the GeneAtlas microarray data set covering whole placental and other human tissues [23] (see Methods for further details). Among the 758 arraybased placenta-enriched genes, 297 were found to be enriched in one of the 3 placental tissues according to our RNA-Seq data, representing a significant overlap between the array and RNA-Seq results $(p=2.2 \mathrm{e}-119$, Fisher's exact test). The difference between the array and RNA-Seq based gene lists could be due to the difference in platforms as well as in tissue samples used for expression profiling. We also used a similar approach to identify tissue-enriched genes in each of the 16 HBM2.0 tissues (15 other HBM2.0 tissues were used as the background). Of all 19 tissues, the three placental tissues were among the tissues with the highest number of tissue-enriched genes, with only testes, brain and white blood cells topping the placental tissues (Figure 2a).

The RNA-Seq data also allowed us to identify genes whose expression was restricted to the placenta (i.e. not a single read detected in any of the 16 non-placental tissues). We identified a total of 170 placenta-specific genes in the three placental compartments combined. We also used the same criteria to identify tissue-specific genes within the 16 HBM2.0 tissues. Consistent with the pattern observed for the tissue-enriched genes, the three placental tissues were among the tissues with the highest number of tissue-specific genes, only after testes and brain. Taken together, these data indicate abundant tissue-specific activation of gene transcription in the placenta.

\section{Genes enriched in or specific to the placenta play important roles in placental function and pregnancy- related diseases}

In order to understand the functional significance of the genes with enriched expression (EE) in the placenta, we asked whether these genes have been implicated in placental biology and/or pregnancy disorders. We compiled two lists of human genes using the Mouse Genome Informatics (MGI) database [24,25] and the PTBGene database [26,27]. The MGI list consisted of human genes whose mouse orthologs are associated with abnormal placental phenotypes when disrupted. The PTB list consisted of genes collected from the literature on genetic association studies on preterm birth (PTB). We found that the placenta-enriched genes overlapped with 70 genes $(19 \%, \mathrm{p}=1.9 \mathrm{e}-9)$ in the MGI list and 20 genes $(24 \%, \mathrm{p}=1.7 \mathrm{e}-5)$ in the PTB list, significantly overrepresented compared to random expectation (Figure $2 \mathrm{~b}$ ).

Many of the genes associated with placental abnormalities in mice (see the heat map of their expression patterns in Figure 2c) were previously known to be involved in physiological and pathological processes related to pregnancy, with examples including prolactin receptor (PRLR) and insulin-like growth factor 2 (IGF2). The PTB list was particularly enriched with interleukin1 (IL1)-related genes, including IL1R1, ILIRN, IL1B, and $I L 1 A$. We also found genes overlapping with both the MGI and PTB lists, such as coagulation factor II (thrombin) receptor $(F 2 R)$ and vascular endothelial growth factor A (VEGFA).

To gain more insight into key processes that may possibly explain functional differences among the three placental tissues, we carried out functional annotation analysis of placenta-enriched genes identified in each of the three placental tissues compared with the other 16 human tissues using DAVID $[28,29]$. The analysis revealed significant enrichment $(\mathrm{p}<0.05$ after Bonferroni correction) of Gene Ontology (GO) terms and KEGG pathways involved in a wide range of biological processes, including focal adhesion, vasculature development, wound healing, and extracellular matrix (ECM)receptor interaction (Table 1). Of particular note is that there was no significantly enriched GO term shared among all three placental tissues, indicating that each compartment of the placenta has its unique profile of active genes involved in different biological processes.

Although there was no GO annotation shared by all three compartments, we identified several biologically relevant enriched categories that overlap between the two membranous compartments amnion and chorion. For example, epithelium development, one of those categories, explains a common compositional feature that exists between the two tissues with both at least partially consisting of a layer of cells that are epithelial in origin (the amniotic epithelium and extravillous cytotrophoblast) [30]. The enrichment of cell/biological adhesion-related genes supports the role of the two membranes as a barrier protecting the fetus from external mechanical force, which requires substantial involvement of cell adhesion molecules. Of note is that we also observed an overrepresentation of mesoderm development in both tissues when we performed our analysis using a different annotation system PANTHER [31,32], 


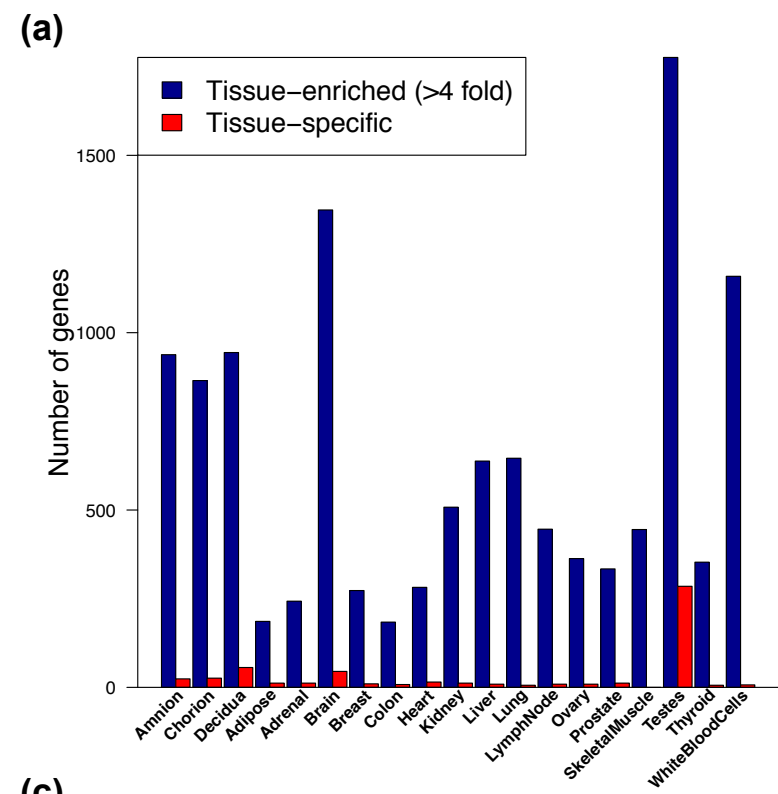

(c)

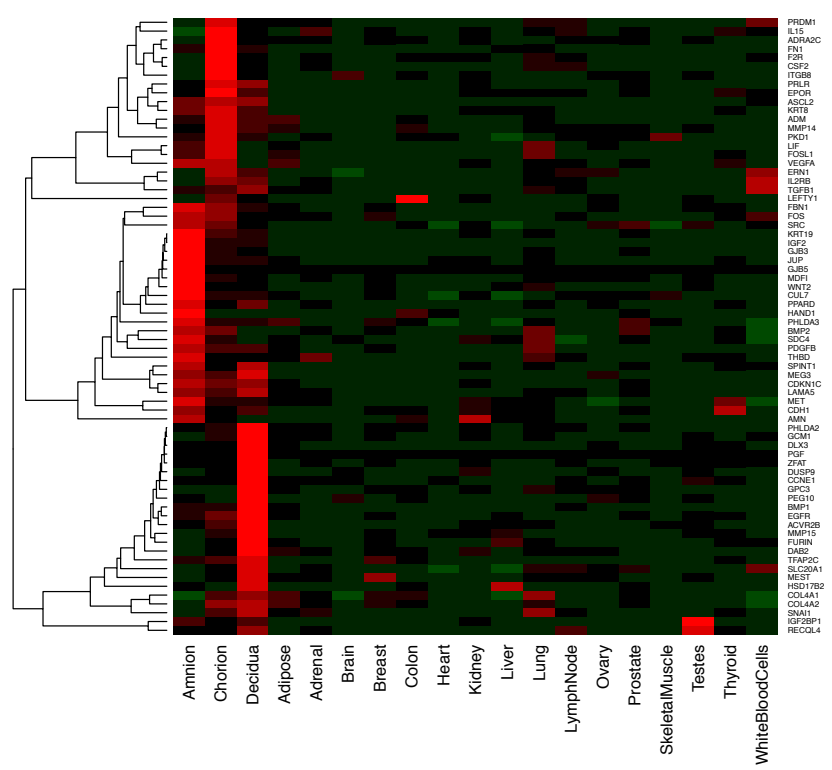

(b)
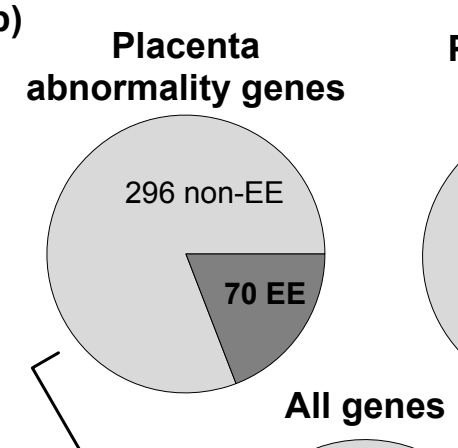

\section{Preterm birth} genes

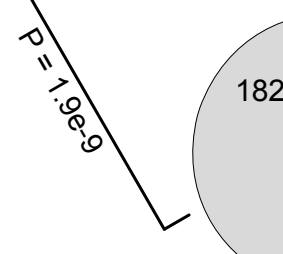

(d)
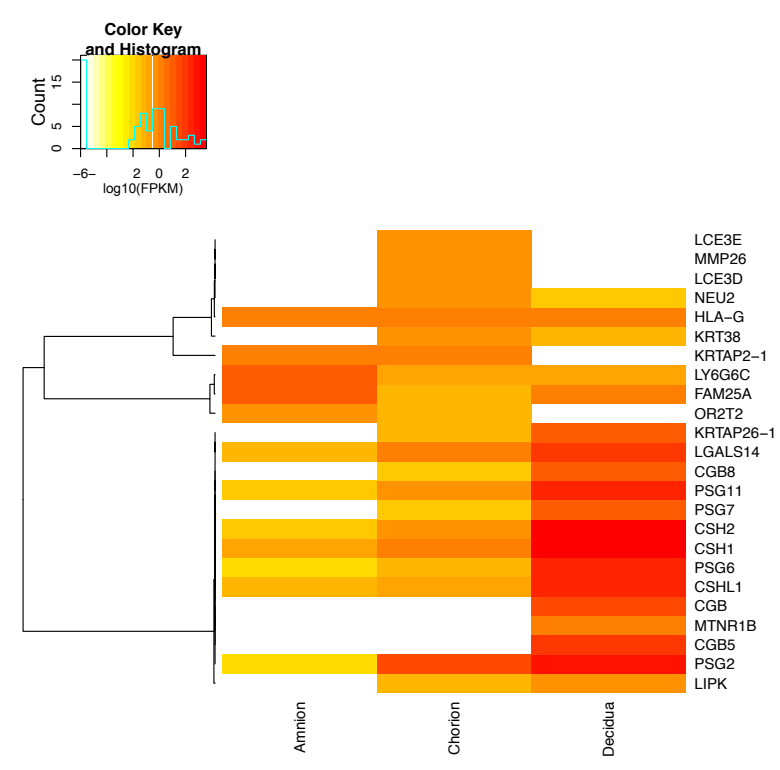

Figure 2 Analysis of placenta-enriched and -specific genes. (a) Number of tissue-enriched (blue bar) and tissue-specific (red bar) genes. Tissue-enriched genes were defined as genes with more than 4-fold change in expression and minimum FPKM of 1. (b) Proportions of overlapping genes between the placenta-enriched gene list and the MGI or PTB gene list (see text and Methods for details). The lighter shade indicates the proportion of non-placentaenriched genes while the darker shade indicates the proportion of placenta-enriched genes. P-values were determined by Fisher's exact test. (c) Expression profile of the 70 placenta-enriched MGl list genes. Gene expression values were normalized for each gene and color-coded using the same scheme depicted in Figure 1. (d) Expression patterns of placenta-specific genes in amnion, chorion, and decidua. Color scheme is based on log10(FPKM value).

which reflects a common structural feature shared by the two membranes.

Among the non-overlapping GO terms, it was noted that there was significant overrepresentation of vascularrelated GO terms such as blood vessel development, vasculature development, blood vessel morphogenesis, and angiogenesis in the chorion, while these terms were absent from the amnion, an avascular tissue. One of the genes belonging to these categories is VEGFA, which is an extensively studied gene that acts as a signal triggering the induction of angiogenesis [33] and has been implicated in pregnancy complications [34-36].

We found that three GO terms are significantly enriched for the decidua with female pregnancy being 
Table 1 Gene Ontology (GO) analysis of placenta-enriched genes

\begin{tabular}{|c|c|c|c|c|c|c|}
\hline Tissue & Category $^{\#}$ & Term & $\begin{array}{l}\text { Number of } \\
\text { genes }\end{array}$ & P-Value & $\begin{array}{l}\text { Fold } \\
\text { Enrichment }\end{array}$ & $\begin{array}{l}\text { Bonferroni- } \\
\text { corrected P-Value }\end{array}$ \\
\hline Amnion & GO_BP & GO:0007398 ectoderm development & 46 & $9.95 \mathrm{E}-21$ & 5.3 & $2.51 \mathrm{E}-17$ \\
\hline Amnion & GO_BP & GO:0008544 epidermis development & 43 & $1.28 \mathrm{E}-19$ & 5.4 & $3.23 \mathrm{E}-16$ \\
\hline Amnion & GO_BP & GO:0007155 cell adhesion & 76 & $1.71 \mathrm{E}-12$ & 2.4 & $4.31 \mathrm{E}-09$ \\
\hline Amnion & GO_BP & GO:0022610 biological adhesion & 76 & $1.85 \mathrm{E}-12$ & 2.4 & 4.67E-09 \\
\hline Amnion & GO_BP & GO:0030855 epithelial cell differentiation & 27 & $1.46 \mathrm{E}-10$ & 4.5 & $3.68 \mathrm{E}-07$ \\
\hline Amnion & GO_BP & GO:0009913 epidermal cell differentiation & 19 & $2.80 \mathrm{E}-10$ & 6.5 & 7.07E-07 \\
\hline Amnion & GO_BP & GO:0030216 keratinocyte differentiation & 18 & 4.05E-10 & 6.8 & $1.02 \mathrm{E}-06$ \\
\hline Amnion & GO_BP & GO:0060429 epithelium development & 33 & 7.05E-09 & 3.2 & $1.78 \mathrm{E}-05$ \\
\hline Amnion & GO_BP & GO:0018149 peptide cross-linking & 10 & $1.14 \mathrm{E}-06$ & 8.5 & $2.87 \mathrm{E}-03$ \\
\hline Amnion & GO_BP & GO:0031424 keratinization & 10 & $1.53 \mathrm{E}-05$ & 6.4 & $3.79 \mathrm{E}-02$ \\
\hline Amnion & GO_BP & GO:0043062 extracellular structure organization & 22 & $1.86 \mathrm{E}-05$ & 2.9 & 4.59E-02 \\
\hline Amnion & GO_BP & GO:0030198 extracellular matrix organization & 17 & $1.91 \mathrm{E}-05$ & 3.6 & 4.69E-02 \\
\hline Amnion & KEGG & hsa04512:ECM-receptor interaction & 21 & $5.92 \mathrm{E}-10$ & 5.4 & $8.05 \mathrm{E}-08$ \\
\hline Amnion & KEGG & hsa04510:Focal adhesion & 28 & $2.30 \mathrm{E}-07$ & 3.1 & $3.13 \mathrm{E}-05$ \\
\hline Chorion & GO_BP & GO:0007155 cell adhesion & 73 & $1.33 \mathrm{E}-11$ & 2.3 & $3.43 \mathrm{E}-08$ \\
\hline Chorion & GO_BP & GO:0022610 biological adhesion & 73 & $1.46 \mathrm{E}-11$ & 2.3 & 3.77E-08 \\
\hline Chorion & GO_BP & $\begin{array}{l}\text { GO:0007166 cell surface receptor linked signal } \\
\text { transduction }\end{array}$ & 123 & 1.09E-09 & 1.7 & $2.81 \mathrm{E}-06$ \\
\hline Chorion & GO_BP & GO:0007398 ectoderm development & 30 & 4.77E-09 & 3.6 & $1.23 \mathrm{E}-05$ \\
\hline Chorion & GO_BP & $\begin{array}{l}\text { GO:0007223 Wnt receptor signaling pathway, calcium } \\
\text { modulating pathway }\end{array}$ & 11 & $9.29 \mathrm{E}-09$ & 11.3 & $2.40 \mathrm{E}-05$ \\
\hline Chorion & GO_BP & GO:0008544 epidermis development & 28 & $1.35 \mathrm{E}-08$ & 3.6 & $3.49 \mathrm{E}-05$ \\
\hline Chorion & GO_BP & GO:0007565 female pregnancy & 21 & 9.50E-08 & 4.2 & $2.46 \mathrm{E}-04$ \\
\hline Chorion & GO_BP & GO:0009611 response to wounding & 51 & $3.61 \mathrm{E}-07$ & 2.2 & $9.33 \mathrm{E}-04$ \\
\hline Chorion & GO_BP & GO:0051270 regulation of cell motion & 27 & 7.39E-07 & 3.0 & $1.91 \mathrm{E}-03$ \\
\hline Chorion & GO_BP & GO:0001501 skeletal system development & 36 & $1.72 \mathrm{E}-06$ & 2.4 & 4.43E-03 \\
\hline Chorion & GO_BP & GO:0060429 epithelium development & 28 & $2.25 \mathrm{E}-06$ & 2.8 & $5.79 \mathrm{E}-03$ \\
\hline Chorion & GO_BP & GO:0001568 blood vessel development & 30 & $2.29 \mathrm{E}-06$ & 2.7 & $5.91 \mathrm{E}-03$ \\
\hline Chorion & GO_BP & GO:0030334 regulation of cell migration & 24 & $2.62 \mathrm{E}-06$ & 3.1 & $6.76 \mathrm{E}-03$ \\
\hline Chorion & GO_BP & GO:0016055 Wnt receptor signaling pathway & 21 & $3.00 \mathrm{E}-06$ & 3.4 & $7.73 \mathrm{E}-03$ \\
\hline Chorion & GO_BP & GO:0001944 vasculature development & 30 & $3.75 E-06$ & 2.6 & $9.65 \mathrm{E}-03$ \\
\hline Chorion & GO_BP & GO:0042127 regulation of cell proliferation & 64 & $6.58 \mathrm{E}-06$ & 1.8 & 1.69E-02 \\
\hline Chorion & GO_BP & GO:0035295 tube development & 27 & $7.56 \mathrm{E}-06$ & 2.7 & $1.94 \mathrm{E}-02$ \\
\hline Chorion & GO_BP & GO:0048514 blood vessel morphogenesis & 26 & $1.06 \mathrm{E}-05$ & 2.7 & $2.71 \mathrm{E}-02$ \\
\hline Chorion & GO_BP & GO:0001525 angiogenesis & 21 & $1.14 \mathrm{E}-05$ & 3.1 & $2.92 \mathrm{E}-02$ \\
\hline Chorion & KEGG & hsa04060:Cytokine-cytokine receptor interaction & 38 & $3.89 \mathrm{E}-10$ & 3.1 & $5.48 \mathrm{E}-08$ \\
\hline Chorion & KEGG & hsa04512:ECM-receptor interaction & 19 & $7.54 \mathrm{E}-08$ & 4.6 & $1.06 \mathrm{E}-05$ \\
\hline Chorion & KEGG & hsa04340:Hedgehog signaling pathway & 14 & $1.99 \mathrm{E}-06$ & 5.1 & 2.80E-04 \\
\hline Chorion & KEGG & hsa05217:Basal cell carcinoma & 13 & 9.93E-06 & 4.8 & $1.40 \mathrm{E}-03$ \\
\hline Chorion & KEGG & hsa04510:Focal adhesion & 25 & 2.91E-05 & 2.6 & 4.09E-03 \\
\hline Chorion & KEGG & hsa05200:Pathways in cancer & 33 & 9.39E-05 & 2.1 & $1.32 \mathrm{E}-02$ \\
\hline Chorion & PANTHER & P00034:Integrin signalling pathway & 26 & $5.49 \mathrm{E}-04$ & 2.0 & 4.40E-02 \\
\hline Decidua & GO_BP & GO:0007565 female pregnancy & 27 & $4.48 \mathrm{E}-12$ & 5.2 & $1.20 \mathrm{E}-08$ \\
\hline Decidua & GO_BP & GO:0042060 wound healing & 26 & $2.91 \mathrm{E}-06$ & 2.9 & 7.77E-03 \\
\hline Decidua & GO_BP & GO:0048732 gland development & 20 & $1.55 \mathrm{E}-05$ & 3.2 & $4.08 \mathrm{E}-02$ \\
\hline
\end{tabular}

Analysis was performed on genes identified as being enriched in each of the three placental tissues compared to the other 16 human tissues

\# GO term Biological Process categories are based on GO FAT definition from the DAVID website; KEGG and PANTHER are the corresponding pathways defined in the DAVID website. 
the most enriched category, consistent with the role of decidua as a principal source of hormones and cytokines pivotal in the maintenance of pregnancy. It was noted that many of the genes associated with female pregnancy have also been implicated in pregnancy-related disorders. These genes include transforming growth factor beta 1 (TGFB1) and placental growth factor (PGF) in $\mathrm{PE}$ [37-39] and corticotropin releasing hormone $(C R H)$ in preterm labor or delivery $[40,41]$.

For placenta-specific genes, we further removed genes with extremely low FPKM values $(<0.3)$ in the placental tissues, which could represent genes with universal low expression in all tissues but sampled by RNA-Seq in the placenta by chance. This led to a final set of 24 placenta-specific well-annotated protein-coding genes with FPKM > 0.3 in at least one placental tissue. The placenta-specific genes are highly enriched for genes encoding pregnancy-related hormones, including pregnancy-specific glycoproteins (PSGs), chorionic somatomammotropin hormones (CSHs), and chorionic gonadotropin, beta polypeptides (CGBs) (Figure 2d).

\section{Expression profiles of splicing factors (SFs) in placental and other human tissues}

The deep RNA-Seq data also allowed us to go beyond whole transcript level changes, to identify transcript isoform changes due to pre-mRNA alternative splicing (AS). Splicing factors (SFs) are RNA binding proteins that play key roles in AS regulation [14]. Tissue- and cell-type specific expression of SFs is a major mechanism that drives AS differences among human tissues [42]. For example, brain-specific SFs NOVA1, NOVA2, and FOX1 control a large number of brain-specific AS events [43]. The epithelial-specific splicing factor ESRP1 is transcriptionally silenced during the epithelial-tomesenchymal transition, which flips the switch off for a genome-wide epithelial splicing regulatory network [44].

To identify SFs with a placenta-specific increase or decrease in expression levels, we compiled a list of sixty well-studied SFs $[14,45]$, and analyzed their RNA-Seq FPKM gene expression levels in the placenta and 16 other human tissues. Hierarchical clustering of the 60 SFs revealed a sub-cluster among the three placental compartments, (Figure 3a), consistent with the clustering pattern based on all genes (Figure 1). This cluster analysis recapitulated the known tissue-specific expression patterns of SFs, such as the brain-specific expression of NOVA1, NOVA2, FOX1 (also known as A2BP1), and BRUNOL4. Interestingly, we identified several SFs with compartment-specific changes in expression levels in the placenta, most notably ESRP1 (in amnion) and MBNL3 (in decidua) (Figure 3b), which we confirmed by qRT-PCR (Figure S2 in Additional file 1). ESRP1 and MBNL3 are known to regulate splicing of a large number of genes in epithelial cells [46] and during myogenic differentiation [47], suggesting a unique set of AS events in individual placental compartments downstream of these master splicing regulators. We also identified several ubiquitously expressed SFs with a significant difference in expression levels among the three placental compartments. For example, FOX2 (also known as RBM9), an important splicing regulator in the heart, muscle, and neurons [14], was expressed two-fold higher in amnion compared to chorion and decidua. Together, the expression profiles of SFs suggest tissuespecific regulation of AS between the placenta and other tissues and between different compartments of the placenta.

\section{RNA-Seq and RT-PCR analysis of exon skipping events in placental and other human tissues}

To directly identify AS differences between the placenta and other human tissues, we calculated the exon inclusion level $(\Psi)$ of alternatively spliced cassette exons in each tissue using RNA-Seq reads that are uniquely mapped to the upstream, downstream, and skipping exon-exon junctions of alternatively spliced exons as previously described [13]. We used a Bayesian approach MATS (Multivariate Analysis of Differential Splicing) [48] to perform pairwise comparisons of tissue pairs to test if the difference in $\Psi$ of any alternatively spliced exon between two tissues exceeds 10\% (see Methods for details). Between the three compartments of the placenta, approximately $0.1 \%$ of exons were found to be differentially spliced (FDR $<0.1$ ). In contrast, there was a much greater degree of splicing difference between placental and other human tissues, with $1.6 \%$ of exons, on average, being differentially spliced between one of the placental tissues and one of the 16 HBM2.0 tissues (Figure 4a). It should be noted that given the moderate sequencing depth of 50-83 million reads per tissue, this analysis is expected to have an appreciable level of false negatives. The true extent of splicing differences among these tissues could be considerably larger.

In order to boost the power of RNA-Seq splicing analysis and obtain a robust set of splicing differences between the placental and non-placental tissues, we pooled the RNA-Seq data of all HBM2.0 tissues. We then compared the pooled data to that of each placental tissue. We identified 393, 637, and 402 differentially spliced exons (in 275, 464, and 289 genes) when comparing the pooled non-placental tissues to amnion, chorion, and decidua, respectively (Figure 4b). 129 exons (in 76 genes) were shared among the three placental tissues. On the other hand, the majority (74\%) of differentially spliced exons identified were restricted to only one of the three placental tissues as compared to the non-placental tissues (Figure 4b). Importantly, among the 744 


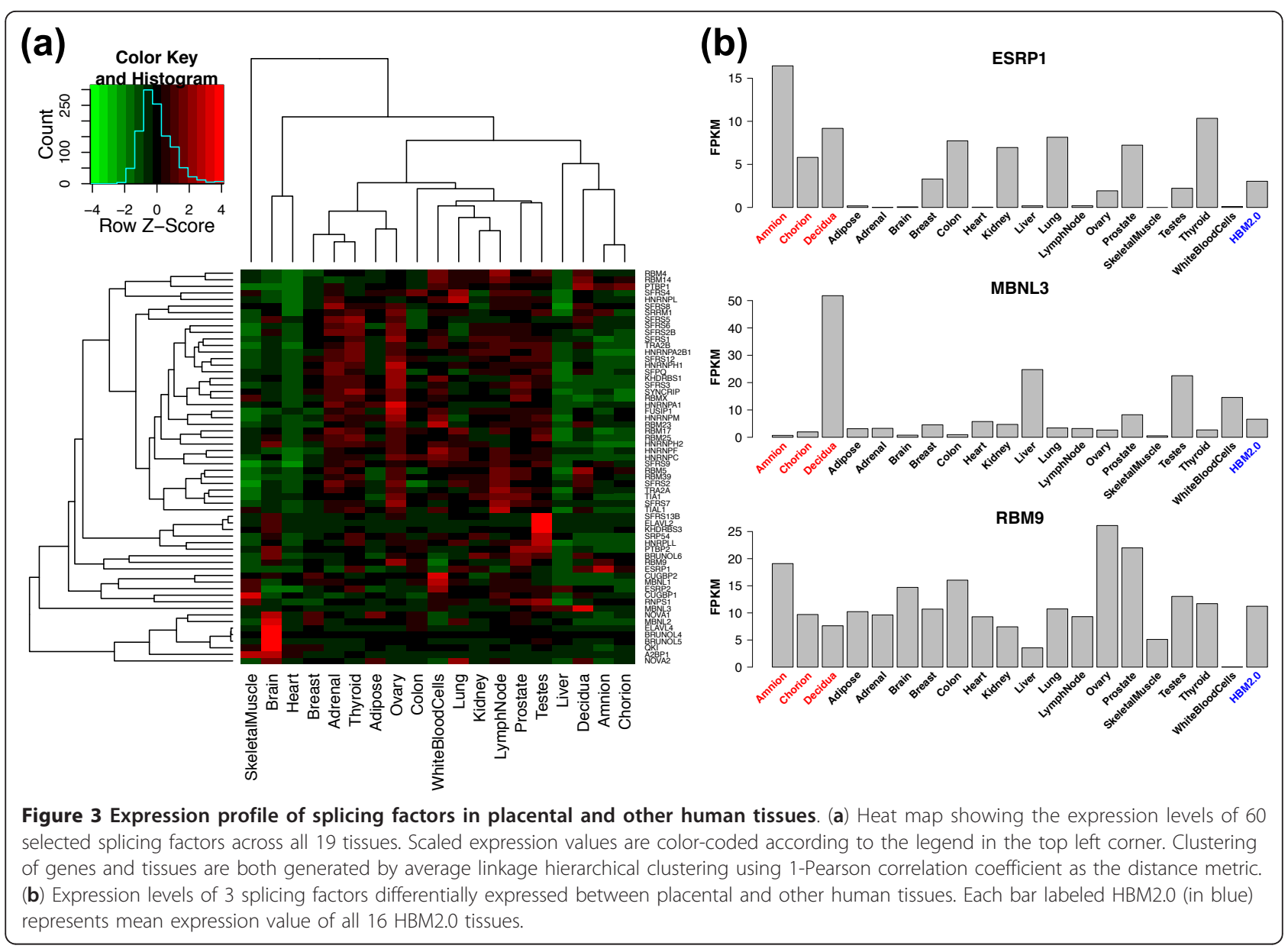

genes containing differentially spliced exons between placental and non-placental tissues, we observed a significant enrichment for genes in the MGI list $(2.8 \%$ over $1.4 \%$ for the genome background, $\mathrm{p}=0.001$ based on Fisher's exact test), indicating the importance of tissuespecific AS in placental function and development. For example, one of these exons (ENSE00000882762) was in integrin, alpha 6 (ITGA6), which forms heterodimers with other integrin components and plays a crucial role in cell adhesion and migration [49,50]. We observed a high inclusion level of this exon in amnion and chorion compared to most of the other tissues, with close to $100 \%$ exon inclusion in amnion as validated by fluorescently labeled RT-PCR (Figure 5a). Exon (ENSE00001385284) in another integrin gene ITGB4 was frequently skipped in the placental tissues (Figure 5b). TCIRG1 (T-cell, immune regulator 1, ATPase, $\mathrm{H}+$ transporting, lysosomal V0 subunit A3) is another differentially spliced gene with multiple known isoforms produced by AS [51,52]. As shown in Figure 5c, the inclusion level of one of its exons (ENSE00000736978) was significantly lower in amnion.
To further confirm the RNA-Seq results of exon splicing, we randomly selected 34 exons in total (including the 3 aformentioned exons) for fluorescently labeled RT-PCR. Using an independent set of term placental samples $(N=4)$ that were not used in the RNA-Seq experiments, we validated the predicted differential splicing events of 27 exons, yielding a validation rate of $79 \%$. The RNA-Seq difference in exon inclusion levels between the placental tissues and the pooled non-placental tissues strongly matched the RT-PCR results (Pearson's correlation coefficient $=0.78$ ) (Figure 6a).

\section{The splicing factor ESRP1 regulates tissue-specific splicing} in amnion

The placenta-specific increase in the expres levels of certain master splicing regulators such as ESRP1 and MBNL3 raises the possibility that downstream exon targets of these regulators may have altered splicing activities in the placenta over non-placental tissues. To test this, we studied the splicing factor ESRP1, which had 5.4 fold higher expression in amnion over the average of the 16 HBM2.0 tissues (Figure 4b). Of note, among the 


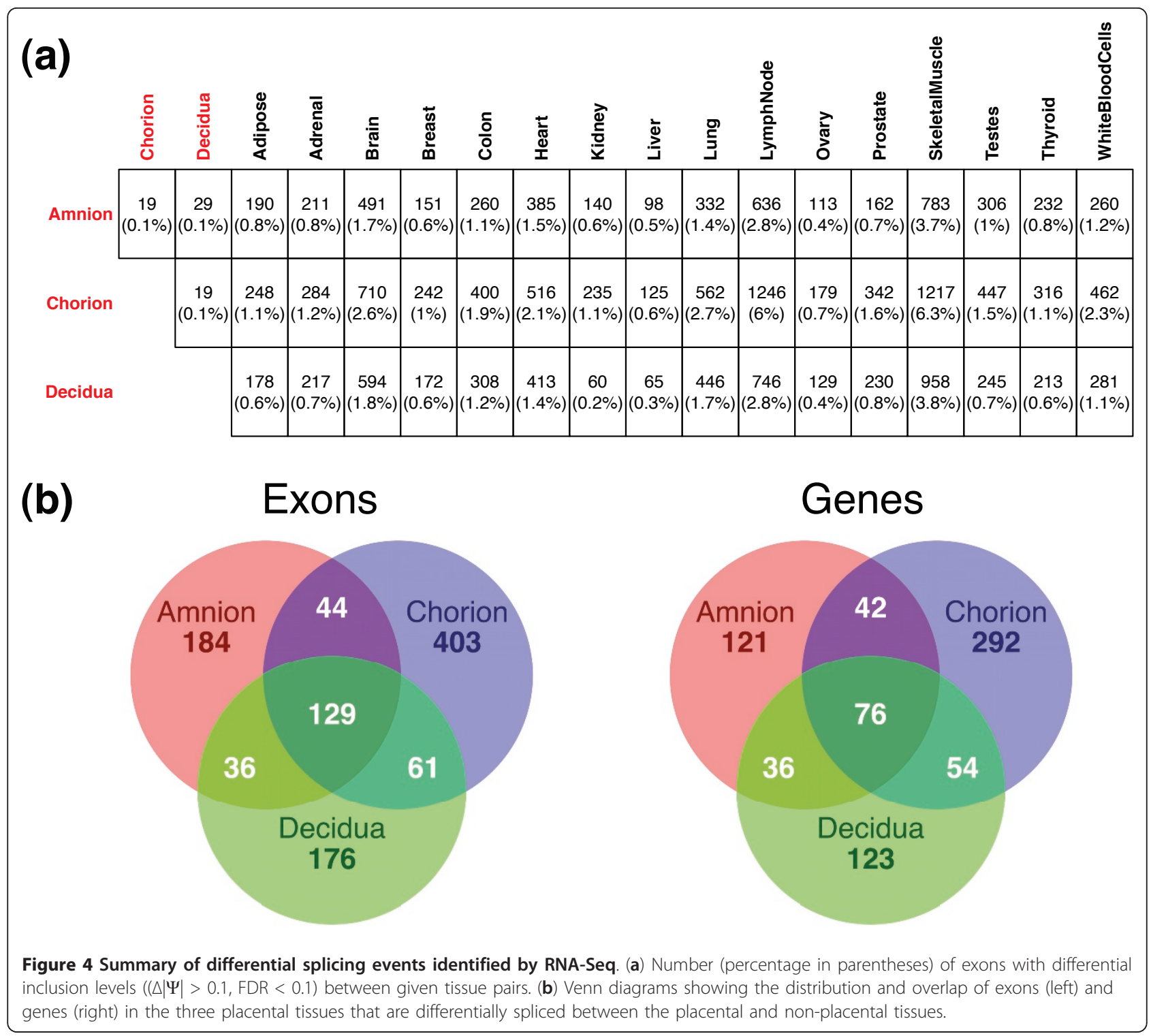

exons validated by RT-PCR as differentially spliced between amnion and non-placental tissues, several were known ESRP1 targets (such as those in ITGA6, LAS1L, MAP3K7, LRRFIP2 and KIF13A; see Figure 6a). To assess the overall enrichment of ESRP1 target exons among differentially spliced exons in amnion, we collected 167 RT-PCR validated ESRP1 target exons from our previous genome-wide analysis of ESRP1-regulated splicing events in epithelial and mesenchymal cells [46]. Of the 167 known ESRP1 target exons, 131 were expressed and detectable in our data. Among them, a significantly enriched set of 20 exons exhibited differential splicing in amnion compared to other human tissues according to RNA-Seq data (Fisher's exact test, $\mathrm{p}=4.3$ e-33) (Figure 6b).
Given our moderate sequencing depth in the placental tissues, it is possible that additional ESRP1 target exons with differential splicing in amnion were missed by RNA-Seq. We therefore selected additional 21 ESRP1 target exons besides the aforementioned 5 validated exons for RT-PCR analysis, resulting in 26 exons tested in total. Seven of those exons did not have any RNASeq reads presumably due to their relatively low expression levels and the limited coverage depth of our sequencing data. We confirmed that 12 of the 26 ESRP1 target exons showed more than 10\% changes in splicing in amnion, with known ESRP1-enhanced exons having increased splicing activities, and known ESRP1-silenced exons having decreased splicing activities. One of the validated ESRP1 target exons was in misshapen-like 


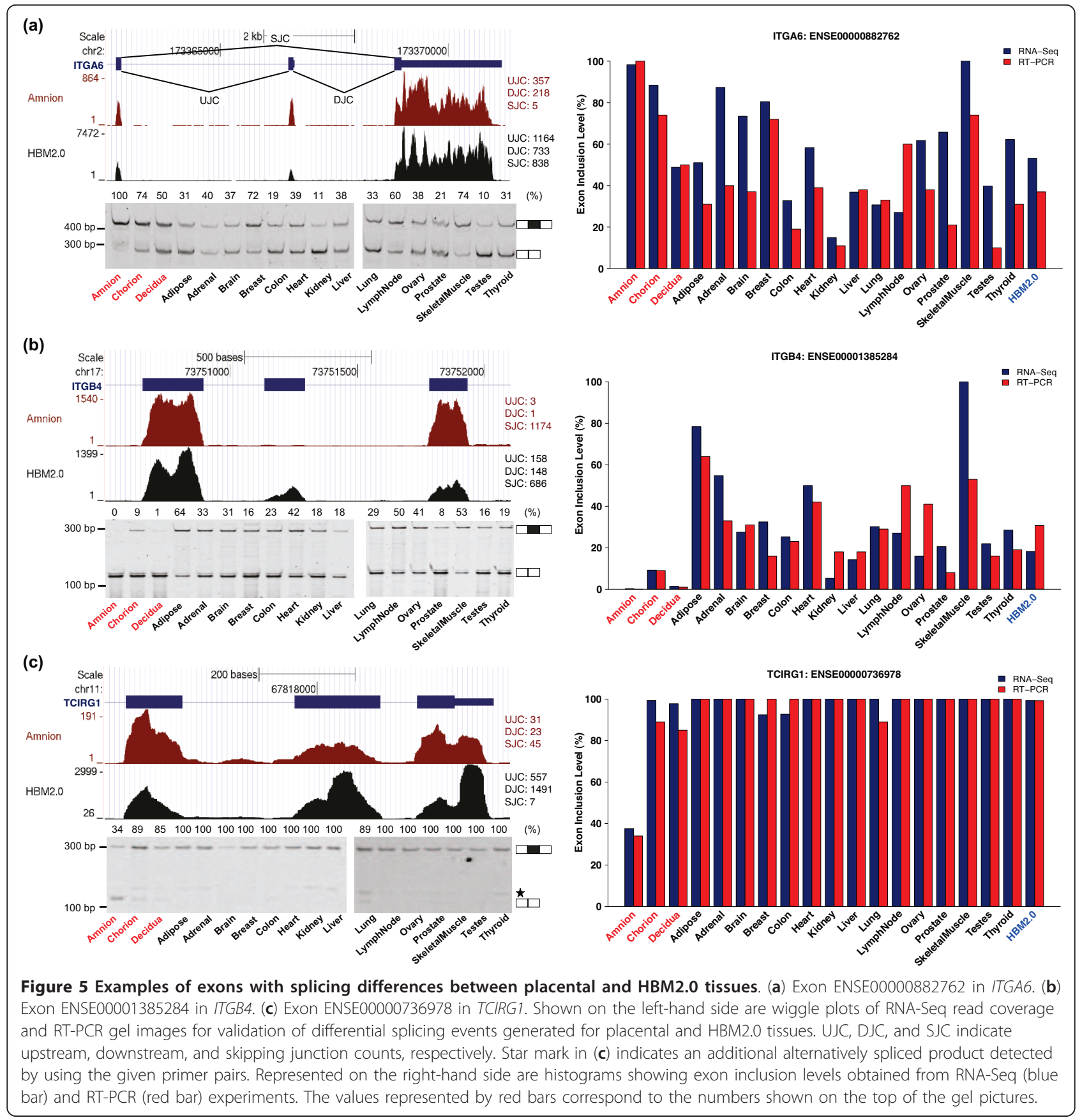

kinase 1 (MINK1), which has an important role in cell adhesion and motility [53]. The exon (ENSE00001213221) in MINK1, a known ESRP1 target had an inclusion level of $>90 \%$ in amnion, approximately $20-30 \%$ higher than those observed for other human tissues (Figure 6c). The increased splicing activity of this MINK1 exon was consistent with the previous observation that ESRP1 positively regulates the splicing of this exon [46].
Analysis of pathways influenced by tissue-enriched expression and differential splicing in placenta

The differential gene- and exon-level expression patterns observed between the placental and non-placental tissues may underlie gene pathways that have key roles in the normal biology of the placenta. To identify pathways and molecular networks influenced by placenta-specific gene expression and splicing, we constructed functional interaction (FI) networks [54] covering genes with 


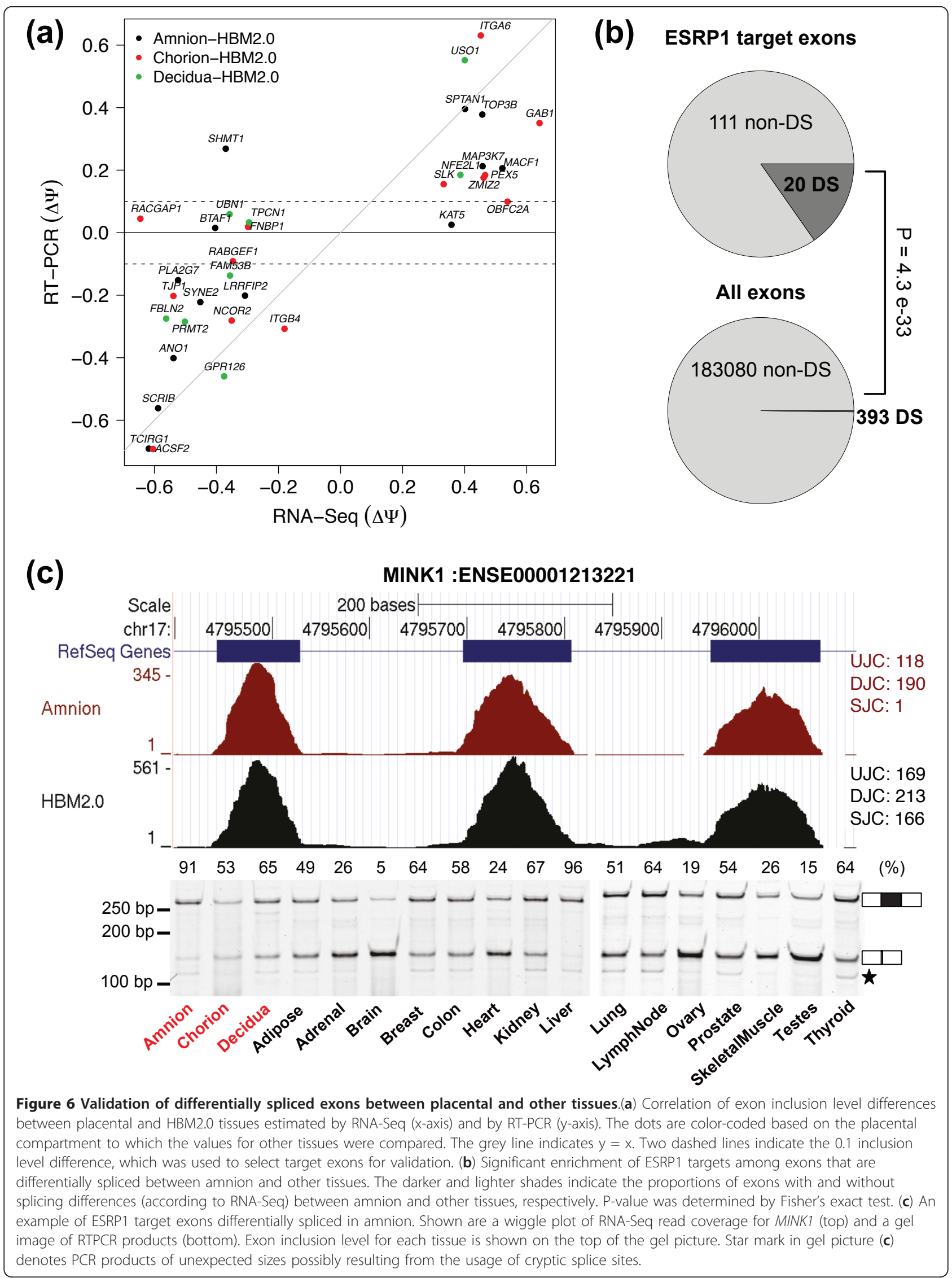


enriched expression (EE) and genes with differential splicing (DS) in amnion, chorion and decidua compared to other human tissues. These genes were used as query sets and projected onto a functional interaction network of human genes constructed from diverse genomic data sources [54]. We used the edge betweenness algorithm [55] to find functional modules in the network, each of which contained enriched functional annotation terms (pathways) that describe the biological roles of genes that are grouped together.

The results of our analysis performed on each of the three placental tissues showed significant enrichment of many functional pathways (Table S3 in Additional file 2 ), including those involved in the regulation of SMAD2/3 signaling, TGF-beta receptor signaling, and HIF-1 alpha TF network, which were significantly overrepresented in module 0 of all the amnion, chorion, and decidua FI networks (shown in Figure 7 is module 0 of the chorion FI network).

The analysis performed on genes abundantly expressed and/or differentially spliced in all three placental tissues revealed strong overrepresentation of pathways related to integrin signaling and focal adhesion (Figure S3 in Additional file 1). These pathways were enriched with genes encoding collagens (COL17A1, COL7A1, COL5A1), laminins (LAMA3, LAMA5), filamins (FLNC, FLNA), integrin (ITGB4), and actinin (ACTN1), all of which are structural components of extracellular matrix (ECM). These results suggest the critical role of ECM in processes involved in normal placental biology. It is interesting to note that the network module contained an appreciable number of both differentially expressed and differentially spliced genes, suggesting that AS and gene transcription act in a coordinated manner to control the overall pathway activity in the placenta.

\section{Novel transcriptional active regions (TARs)}

One major advantage of RNA-Seq compared to microarray technology is its capability to detect un-annotated novel transcripts. To identify novel transcriptional active regions (TARs) in placental tissues, we used the software Scripture [56] for $a b$ initio reconstruction of transcripts for each tissue after sequence mapping with Tophat [21] (see details in Methods). We identified approximately 100,000 transcripts in each of the placental tissues with more than $70 \%$ of them being multiexon transcripts (Table 2). To reduce false signals, only multiexon transcripts were used in the following analysis. After overlapping transcripts were merged into one single TAR, a total of $13,469,16,987$, and 15,158 TARs were found in amnion, chorion, and decidua, respectively. We filtered out the ones overlapping with the annotated transcripts from the NCBI RefSeq, UCSC, Ensembl, and Vega database and identified 604, 1,007, and 896 novel TARs in amnion, chorion, and decidua, respectively. The expression levels of the identified novel TARs are listed in Table S4 in Additional file 3. Importantly, a large proportion of these novel TARs $(285,456$, and 468 in the corresponding placental tissues) are placenta-specific or more than 4 fold enriched compared to non-placental tissues. Shown in Figure 8 is one example of novel TARs on chromosome 16 (chr16:50424807-50430893) expressed in amnion with a high FPKM value of 7.1. Of note, this transcript is not documented in any human gene databases, although the existence of human expressed sequence tags (ESTs) at this locus further supports the validity of this TAR (Figure 8).

We also used RNA-Seq data to identify novel exons in annotated genes. There are a total of between 93 and 103 thousand exons identified in the TARs overlapping with annotated genes. Although more than $80 \%$ of these exons were well annotated with the same 5' and 3' ends, we detected between 494 and 585 totally new exons with no sequence overlap with any annotated exons in the placental tissues. These novel TARs and exons provide a valuable resource for novel transcripts with potential functional significance in the placenta.

\section{Discussion}

With the emergence of new high-throughput technologies such as RNA sequencing, we have recently witnessed a remarkable increase in our knowledge of mammalian transcriptome content and diversity. There has been a particular surge in our understanding of the transcriptome diversity between different tissues and cell types. For example, Wang et al. performed an RNA-Seq analysis of 15 human tissues and cell lines and identified over 22,000 tissue-specific AS events [13]. Other studies have established the association between tissue-specific expression of SFs and genome-wide changes in tissuespecific splicing patterns $[42,45]$, which underscores a critical role of AS regulation in tissue differentiation and specialization.

The majority of previous gene expression studies of human placental tissue have only provided gene-level insights [6-10], driving the need for higher-resolution analysis to enable a better understanding of the complexity of the placental transcriptome at the level of exon splicing. AS, which has a well-established role in cell differentiation $[57,58]$, may be critical for the proper functioning of the placenta, an organ composed of a variety of differentiated cell types, each with its own specific functions during pregnancy. Thus, uncovering the complexity of AS in the placental transcriptome will provide a valuable basis for understanding genes with functional and clinical relevance in placental biology and pathophysiology. 


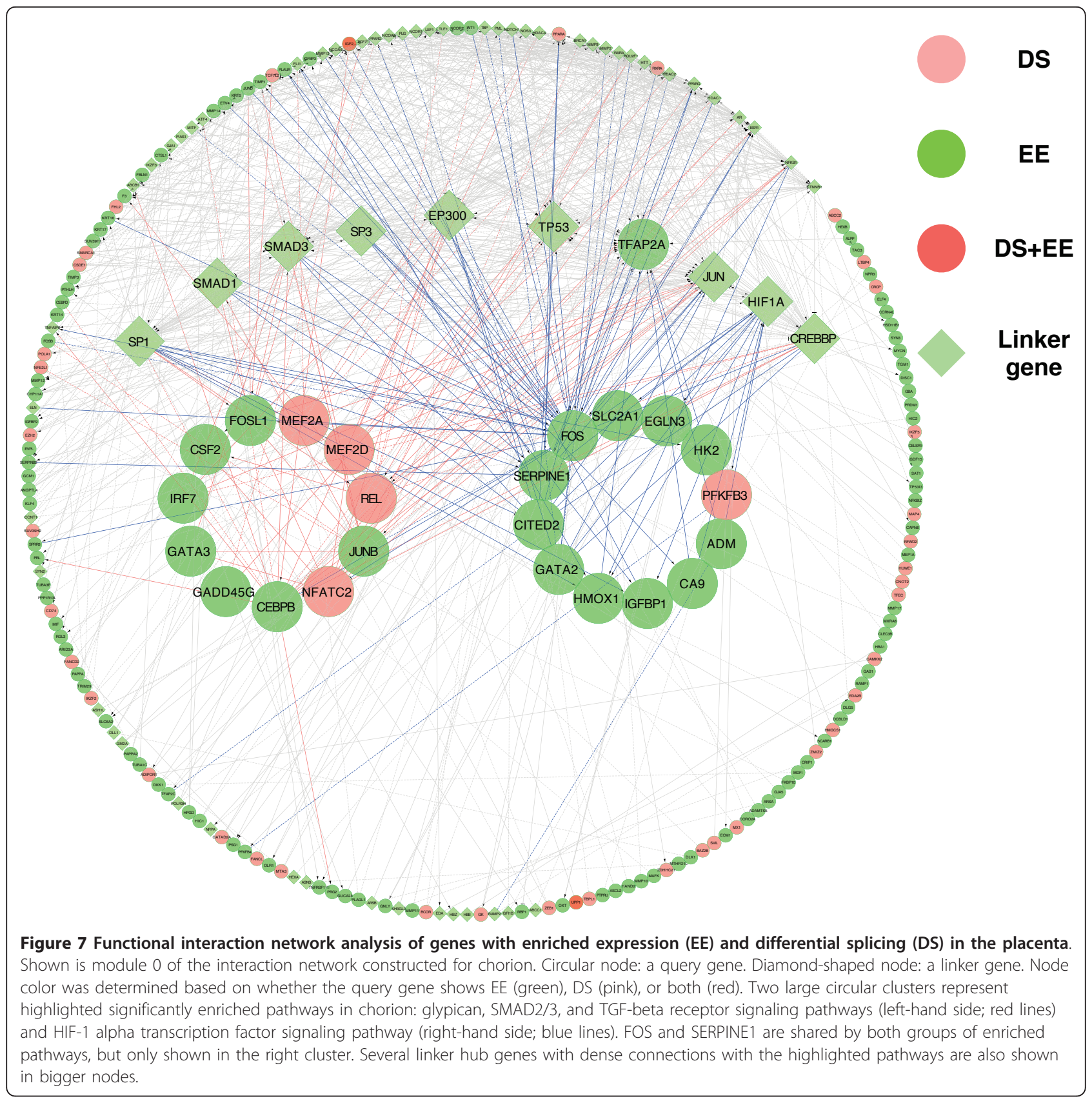

In the present study, we used RNA-Seq to characterize the transcriptome of selected compartments of the human placenta from normal term pregnancies. RNASeq allows an unbiased and sensitive interrogation of the full repertoire of placental mRNA transcripts. We took a two-step approach to analyze the RNA-Seq data at both the gene-level and the exon-level. First, we investigated differential gene expression between the placental and other human tissues to identify genes that are specifically or abundantly expressed in the placenta. Second, we carried out exon profiling as well as SF expression profiling to find AS events and their potential regulators that are differentially present in the placental versus non-placental tissues.

We have compared placenta-enriched genes to genes with putative functional significance in the placenta using the mouse phenotype data and human PTB association study data. We observed that genes implicated in placental abnormalities and PTB are enriched among the genes with placenta-enriched expression profiles. We note that the mouse phenotype data from MGI were generated independent of any previously known 
Table 2 Novel transcriptional active regions (TARs) and exons discovered in placental tissues

\begin{tabular}{|c|c|c|c|}
\hline & Amnion & Chorion & Decidua \\
\hline \multicolumn{4}{|l|}{ Transcripts } \\
\hline Total transcripts & 92,265 & 107,371 & 105,158 \\
\hline Multi-exon" ${ }^{\#}$ transcripts & 69,721 & 69,858 & 75,958 \\
\hline Perfect match with annotated transcripts (ignoring transcript start and end) & 21,288 & 22,724 & 25,077 \\
\hline Total TARs* & 13,469 & 16,987 & 15,158 \\
\hline Novel TARs (not overlapping with the combined annotation of Ensembl, UCSC, RefSeq and Vega genes) & 604 & 1,007 & 896 \\
\hline \multicolumn{4}{|l|}{ Internal exons in TARs overlapping annotated transcripts } \\
\hline Total exons & 93,506 & 103,356 & 100,003 \\
\hline Annotated exons & 75,154 & 81,591 & 83,283 \\
\hline Novel exons with one end ( $5^{\prime}$ or $\left.3^{\prime}\right)$ shared with an annotated exon & 16,907 & 20,121 & 15,246 \\
\hline Novel exons overlapping with an annotated exon but with no shared $5^{\prime}$ or $3^{\prime}$ end & 950 & 1,093 & 876 \\
\hline Novel exons not overlapping with any annotated exons & 494 & 537 & 585 \\
\hline
\end{tabular}

Analysis was performed using software Scripture for $a b$ initio reconstruction of transcripts for each placental tissue after sequence mapping with Tophat.

*TAR: Transcriptional Active Region after merging overlapping transcripts

\# Single exon transcripts were not included in all the downstream analysis

gene expression pattern in the placenta. Among such genes are $P R L R$ and $F 2 R$, genes encoding receptors for prolactin and thrombin, respectively, whose levels are precisely regulated during pregnancy $[59,60]$. The enrichment of IL1-related genes was also noted, suggesting the importance of IL1 signaling in normal placental function and pregnancy. IGF2, one of the genes associated with abnormal placental phenotypes in mice, is known for its active role in placental and fetal growth $[61,62]$. Together, these provide a link between highly expressed placenta-enriched genes and their functional importance in the placenta. Similarly, our work provides evidence suggesting the importance of genes uniquely expressed in the placenta in diverse pregnancy-related processes, with examples including $\mathrm{CSH} 1$ in the regulation of fetal growth [63], $C G B$ in the maintenance of early pregnancy $[64,65]$, and human leukocyte antigen-G (HLA-G) in feto-maternal immune tolerance $[66,67]$. In addition, we observed a significant enrichment of differentially spliced genes in the placenta among genes with placental phenotypes in the mouse, suggesting the importance of tissue-specific AS in placental development and function.

Because the HBM2.0 data all came from adult tissues, it is possible that some placenta-enriched genes identified in our study reflect age-specific expression signatures. Because of the unavailability of RNA-Seq data from other fetal tissues, we assessed this possibility using the GeneAtlas array data [23]. There were 4 fetal tissues (brain, liver, lung, and thyroid) included in the

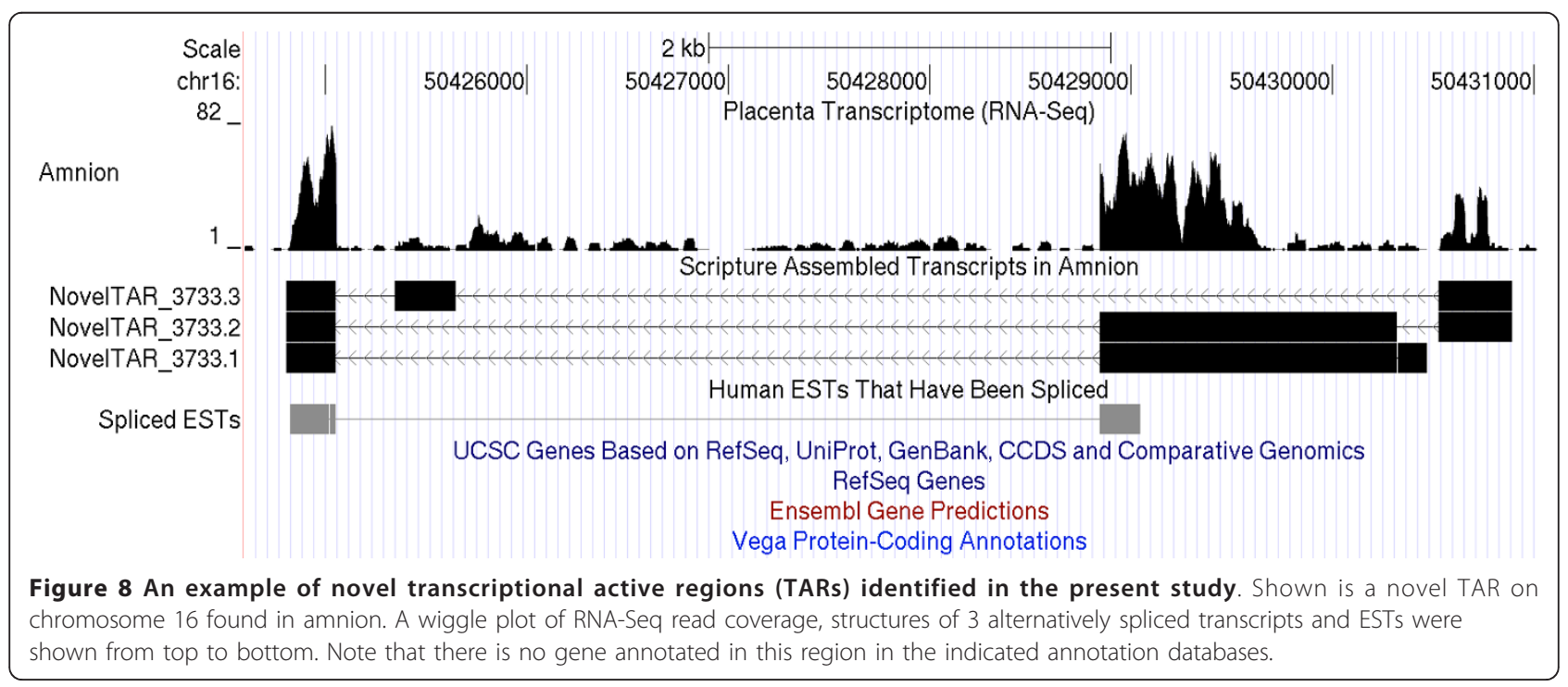


GeneAtlas data. Of the 297 genes with at least 4-fold enrichment in the placenta over adult tissues in both the GeneAtlas array data and our RNA-Seq data, the vast majority (281 genes) were more than 4-fold enriched in the placenta compared with the 4 fetal tissues according to the GeneAtlas array data. This suggests that the placenta-enriched genes identified in our study reflect genuine placenta-associated gene expression signatures. In addition, the strong association of placental expression enrichment with placental diseaserelated gene sets further supports that most of the placenta-enriched genes found here reflect tissue effect rather than age effect.

Given the heterogeneous tissue composition of the placenta, we have characterized the transcriptome profiles of the placenta not only at the whole-organ level, but also at the sub-organ level. It should be noted that the placental samples used in our study (amnion, chorion, and decidua) may not be completely pure, containing minor contamination with other placental components. Nonetheless, our study demonstrated that they are highly enriched for the corresponding tissue types, displaying compartment-specific expression profiles and splicing patterns. The amnion is the innermost layer of the fetal membranes lining the amniotic cavity and is composed of an epithelial cell layer on top of a basement membrane and an avascular matrix [68,69]. Consistent with these histological properties of the amnion, we have detected enrichment of genes involved in cell/focal adhesion and observed that the epithelial splicing regulator ESRP1 was highly expressed. Our splicing analysis of the amnion using RNA-Seq and RTPCR revealed 20 and 12 known ESRP1 target exons, respectively, with differential splicing activities in the amnion. It should be noted that ESRP1 is a master celltype-specific splicing regulator critical for maintaining the epithelial cell identity and has been implicated in a variety of developmental and disease processes [46]. The ESRP1 target exons are strongly enriched in genes involved in the regulation of cell adhesion such as the exon in MINK1 [53], that was found to be differentially spliced in the amnion compared to other human tissues by RNA-Seq and validated by RT-PCR. These data support a role of the ESRP1 splicing regulatory network in the amnion. The chorion, the outer layer of the fetal membranes in contact with the decidua, consists of the reticular layer, the basement membrane, and the trophoblast layer [30]. Similar to the amnion, genes with a role in cell/biological adhesion are also enriched in the chorion, which may be important for the adherence of the trophoblast layer to the decidua [70]. The enrichment of genes involved in vascular-related processes in the chorion may be explained by velamentous vessels traversing the extraplacental membranes or maternal vessels in interdigitating decidua processed along with the chorion. Unlike the two fetal membranes, the decidua is of maternal origin $[1,10]$. It is noteworthy that genes related to female pregnancy were significantly enriched in this compartment of the placenta, further supporting the crucial role of this tissue in pregnancy. Of note, we observed significant differential expression of a splicing factor MBNL3 in the decidua. In future studies, it would be useful to examine how MBNL3 globally impacts gene splicing and function in the decidua.

We also examined potential interactions among genes highly expressed and differentially spliced in the placenta compared to other human tissues by constructing FI networks composed of sub-network modules enriched for specific gene categories and functional pathways. Analysis performed separately on each of the three placental tissues revealed enrichment of set of pathways commonly enriched in all three compartments, for example, regulation of cytoplasmic and nuclear SMAD2/3 signaling and TGF-beta receptor signaling. These pathways are known to be involved in a wide range of cellular processes [71], which reflects the versatile function of the placenta that can be achieved through diverse cellular activities occurring in different parts of the placenta. Among its other main functions, the placenta plays an important role as an immune barrier, protecting the fetus from the mother's immune system [1]. This function is reflected by the enriched expression of transcription factors (TFs) involved in immune regulation such as GATA3 and IRF7 as well as the differential splicing of $R E L$, a member of the Rel/NFKB family and NFATC2, a member of the nuclear factors of activated $\mathrm{T}$ cells transcription complex. HIF-1 alpha TF network is another pathway that was enriched in module 0 of all the three FI networks. The placenta, during its development, is exposed to different oxygen environments and tight regulation of oxygen homeostasis is necessary for proper placental development and function, which requires active involvement of the HIF-1 alpha TF network [72]. These findings suggest: (1) the common importance of these pathways in the functioning of the different parts of the placenta examined in the present study; and (2) the importance of the regulation of gene expression and AS as critical mechanisms underlying anatomical, developmental, and functional specialization of the placenta. When the analysis was performed on all of the tissues combined, we observed the overrepresentation of ECM-related gene sets such as integrin signaling pathway, ECM-receptor interaction, focal adhesion, and integrin cell surface interactions. These results provide evidence for the role of ECM in placental development and placental cell proliferation as demonstrated in earlier studies [73,74]. 


\section{Conclusions}

Our study provides the first comprehensive view of the placental transcriptome at exon-level resolution, and reveals that tissue-specific gene regulation in the placenta involves complex changes in both gene transcription and exon splicing. Our data should serve as a valuable resource for future in-depth investigations into what genes contribute to specification of the placenta. All of the RNA-Seq data can be accessed as the raw RNA-Seq reads and as a processed UCSC Genome Browser custom track http://intron.healthcare.uiowa. edu/placenta/. Furthermore, the findings of this work may provide useful clues on how those genes/pathways, when altered at either the gene level or exon level, could lead to pregnancy-related diseases. Future research using tissues from abnormal conditions will help expand our knowledge of the transcriptome alterations and pathological processes involved in maternal and fetal complications.

\section{Methods}

\section{Tissue collection}

Fresh human placentas were obtained within one hour of normal vaginal delivery at term with signed informed consent under protocols approved by the University of Iowa Institutional Review Board (200506792, 200411759). The placentas were received largely intact when visually inspected. Each placenta was dissected into the fetal (amnion, chorion) and maternal (decidua) portions. The amnion and chorion were taken from the reflected membranes and separated by blunt dissection. Decidual tissue samples were macroscopically isolated from the maternal-facing surface of the placenta. The dissected tissues were cut into small pieces and placed in RNAlater ${ }^{\circledR}$ solution (Applied Biosystems, Foster City, CA). To ensure that our results better reflect the true nature of the normal term placental transcriptome, we used placentas from term ( $\geq 37$ weeks of gestation) deliveries with spontaneous onset of labor.

\section{RNA extraction}

Total RNA was extracted from each tissue using the TRIzol $^{\circledR}$ reagent (Invitrogen, Carlsbad, CA) according to manufacturer's instructions and stored at $-80^{\circ} \mathrm{C}$ until used. For RNA-Seq, we prepared pooled amnion, chorion, and decidua samples, using an identical set of RNA from five different individuals. The pooled samples were of high quality with an RNA integrity number $($ RIN) $>8$. For validation of differential splicing events and splicing factor expression, we generated RNA pools, each for amnion, chorion, and decidua, consisting of 4 biological replicates that are independent from those used in the RNA-Seq experiments.
For validation experiments, we purchased total RNA representing all HBM2.0 tissues except white blood cells from Applied Biosystems (Foster City, CA) or Clontech (Mountain View, CA).

\section{Library construction and sequencing}

Library preparation and paired-end sequencing were performed by Ambry Genetics (Aliso Viejo, CA). Double-stranded cDNA fragments were synthesized from mRNA, ligated with adapters, and size-selected for library construction according to the manufacturer's protocol (Illumina, San Diego, CA). Each of the three libraries generated was loaded onto one lane of the flow cell at $8 \mathrm{pM}$ concentration. Two paired-end runs (72 bp and 54 bp runs) of sequencing were carried out on the Illumina Genome Analyzer IIx. Initial data processing was performed using RTA 1.6.47.1 (SCS version 2.6.26). Sequence quality filtering script was executed in the Illumina CASAVA version 1.6.0 software (Illumina, Hayward, CA).

\section{Sequence alignment}

For each end (forward or reverse) of the paired-end reads from placenta, we trimmed the sequence to $50 \mathrm{bp}$ based on the sequencing error profile. The HBM2.0 data consist of the following tissues: adipose, adrenal, brain, breast, colon, heart, kidney, liver, lung, lymph node, ovary, prostate, skeletal muscle, testes, thyroid and white blood cells. Each tissue came from a single adult donor with ages ranging from 19 to 86. The HBM2.0 data are accessible from EBI ArrayExpress track: http://www.ebi. ac.uk/arrayexpress/browse.html?keywords=E-MTAB-513. For HBM2.0, we used all the $50 \mathrm{bp}$ from the paired end data. Each read was mapped to the reference human genome (hg19) as well as all possible exon-exon junctions (Ensembl genes, r57) as previously described [75]. Each exon-exon junction is 84 bp in length, containing the last $42 \mathrm{bp}$ of the upstream exon and the first $42 \mathrm{bp}$ of the downstream exon. We used Bowtie [76] to map those reads, allowing up to three mismatches and also required that each read has at most three possible mapped locations in either the human genome or all possible exon-exon junctions. For each pair of forward and reverse reads, we enumerated all possible combinations of mapped forward and reverse reads. We required that the two ends from the same read pair should be on the same chromosome but in the opposite orientation. Since $98.4 \%$ of human introns have length less than 50 $\mathrm{kb}$ (data not shown), we also required that the two ends should be within $50 \mathrm{~kb}$ of each other in the mapped genomic locations. Based on these criteria, we collected a set of uniquely mapped pairs to do the subsequent analysis. 
Gene expression quantification using RNA-Seq data

We estimated the gene expression level using RNA-Seq by the Fragments Per Kilobase of gene per Million mapped fragments (FPKM). Ensembl release r57 was used for gene annotation. To avoid the ambiguity of assigning reads to different isoforms of the same gene and obtain a robust estimate of the overall gene expression levels, we used an exon union method by counting all reads mapped to any exon in any of the gene's isoforms. This approach is similar to the original RPKM definition [22] instead of the transcript isoform level estimate as in Cufflinks [21].

\section{Placenta-enriched genes based on GeneAtlas array data}

We selected 16 tissues from the Human GeneAtlas array dataset [23], consisting of whole placental tissue and 15 non-placental tissues. These 15 tissues are identical to those examined in the Human Body Map 2.0 project except that breast tissue is not included in the GeneAtlas data set. We compared the expression values from the whole placental tissue to the average values from 15 other human tissues and generated a list of 758 genes with at least 4-fold enrichment in the whole placenta.

\section{Enrichment of GO functional categories and pathways}

To identify the overrepresented functional categories among the genes with enriched expression or differential alternative splicing in the placenta compared to the 16 HBM2.0 tissues, we used the online functional annotation tool DAVID $[28,29,77]$. All the expressed proteincoding genes in the combined placenta and HBM2.0 data were used as the background. We used the GO_BP_FAT categories for GO biological process categories and KEGG and PANTHER annotation for pathway analysis. A modified Fisher's exact test (EASE score) from DAVID was used for testing the significance of functional category enrichment. The significant categories with a $\mathrm{p}$-value $<0.05$ after Bonferroni correction were reported.

Placental abnormality- and preterm birth-related genes To obtain genes associated with abnormal placental phenotypes, we searched the MGI database [24,25] for 4 MGI phenotypes (abnormal amnion morphology, MP:0005029; abnormal chorion morphology, MP:0002836; abnormal placenta morphology, MP:0001711; abnormal maternal decidual layer morphology, MP:0004256). These mouse genes were mapped to human based on the Human and Mouse Orthology in the MGI database to obtain the orthologous human genes. Preterm birth-related genes were taken from the preterm birth genetics knowledge base PTBGene [26,27], a regularly updated and manually curated collection of genes implicated in published association studies of PTB.

\section{Alternative splicing analysis}

We focused our analysis on the exon-centric analysis. We only used those reads that uniquely mapped to the splicing junctions to estimate the exon inclusion level ( $\Psi$ ) of alternatively spliced exons. We used the same formula as in [13]: $\Psi=I+S$. Suppose $U J C, D J C$, and SJC represent read counts of upstream junction, downstream junction and skipping junction respectively, then junction read counts from the exon-inclusion transcript $(I)$ equal $((U J C+D J C) / 2)$ and read counts from the exonskipping transcript equal $S$. To find the placenta specific exon inclusion/skipping, we also pooled all the reads from the 16 HBM2.0 tissues to get a mean inclusion level of non-placental tissues. Utilizing the read counts information on the 3 types of junctions of each exon, we used a multivariate Bayesian algorithm MATS (Multivariate Analysis of Transcript Splicing) [48]. Briefly, MATS uses a multivariate uniform prior to model the between-sample correlation in exon splicing patterns, and a Markov chain Monte Carlo (MCMC) method coupled with a simulation-based adaptive sampling procedure to calculate the $\mathrm{P}$ value and false discovery rate (FDR) of differential AS. Importantly, the MATS approach provides the flexibility to identify differential AS events that match a given user-defined pattern. Suppose $\Psi 1$ and $\Psi 2$ are the exon inclusion levels of 2 tissues and we want to test if $|\Psi 1-\Psi 2|>10 \%$, we can obtain the Bayesian posterior probability $\mathrm{P}=\mathrm{P}(|\Psi 1-\Psi 2|$ $>10 \%$ ) and subsequent $\mathrm{P}$ value and FDR. The MATS software can be downloaded from http://intron.healthcare.uiowa.edu/mats/.

\section{Fluorescently labeled RT-PCR and qRT-PCR}

We validated 2 sets of exons using RT-PCR. One set includes 34 exons that showed significant differential splicing ( $>10 \%$ inclusion level difference with FDR $<0.1$ between one of the three placental tissues and the pooled HBM2.0 tissues). Another set includes 21 known ESRP1 target exons that are predicted to have differential splicing due to differential expression of ESRP1 in amnion. Single-strand cDNA was synthesized from total RNA using the High Capacity cDNA Reverse Transcription Kit (Applied Biosystems) according to the manufacturer's protocol. Fluorescently labeled RT-PCR was performed as described [78]. Briefly, for each tested exon, we designed a pair of primers targeting flanking constitutive exons. Fluorescent labeling of PCR products was carried out according to a method modified from that of Schuelke [79]. PCR products were separated on a polyacrylamide gel and the fluorescence signal was captured and quantified using a Typhoon 9200 scanner 
(Molecular Dynamics, Sunnyvale, CA) and the Quantity One 4.6.2 software (Bio-Rad, Hercules, CA). To validate the expression levels of ESRP1 and MBNL3, qRT-PCR was performed using the Power SYBR Green PCR Master Mix (Applied Biosystems) and the 7900HT Fast Real-Time PCR System (Applied Biosystems). In each experiment, HPRT1 was used as an endogeneous reference. Three technical replicates were included for each sample. Data were generated using the SDS 2.3 software (Applied Biosystems) and analyzed using the comparative CT method [80]. All primer sequences used for this study and exon inclusion levels from both RNA-Seq and RT-PCR are provided in Table S5 in Additional file 4 and gel pictures are shown in Figure S4 and S5 in Additional file 5 .

\section{Functional interaction networks of genes with placenta- enriched expression or differential splicing}

We combined the genes with placenta-enriched expression or differential splicing into 4 query gene sets: combination of placenta-enriched genes with FPKM > 1 and $>4$ fold enrichment (EE) and genes significantly differentially spliced with FDR $<0.1$ and $|\Psi 1-\Psi 2|>10 \%$ (DS) compared to the HBM2.0 tissues in each of the three placental tissues individually and the intersection set of all three tissues. We projected each of the query gene set onto the functional interaction network of human genes from the Reactome database [54] using the Reactome FI network plug-in in Cytoscape [81]. Edgebetweenness algorithm was used to cluster the network into modules [82]. Pathway enrichment analysis was done on the whole network and within each of the subnetwork modules. The networks from representative modules are visualized in Cystoscape [81]. Enriched pathways with FDR $<0.05$ in modules with size of at least 40 are listed in Table S3 in Additional file 2.

\section{Discovery of novel transcriptional active regions (TARs)}

Scripture software [56] was used for $a b$ initio reconstruction of the transcripts for each tissue after mapping with Tophat [21]. Same as in the expression analyses, reads of the three placental tissues were trimmed at 3' end to $50 \mathrm{nt}$ before mapping. As reported, starts and ends of reconstructed transcripts were usually not as accurate as splice sites, thus single-exon transcripts were removed in the analyses. The reconstructed transcripts were clustered into TARs when there were any overlaps between transcripts. Overlapping between two transcripts was defined when they are in the same strand and have at least one common internal exon boundary, which means that they have at least one common exon start site or exon end site. Novel TARs were determined by comparison with a combination of annotated transcripts from the NCBI RefSeq, UCSC, Ensembl, and
Vega database. A TAR was considered as novel if there is no overlap of TAR with any annotated transcript using the above definition. We also examined the exon distributions within the TARs overlapping with annotated transcripts. Because the start and end of transcript annotations usually vary greatly, to compare the reconstructed exons within TARs overlapping annotated transcripts with the exon annotations, we only focused on the internal exons in our analysis. To compare in all tissues the expression levels of novel TARs identified in placental tissues, we first used the exons identified in the novel TARs, and then calculated FPKM values in the same way as in the analysis of known gene expression for all three placental tissues and 16 HBM2.0 tissues.

\section{Data accessibility}

All data described here can be accessed from: http:// intron.healthcare.uiowa.edu/placenta/.

\section{Additional material}

Additional file 1: Tables S1 and S2 and Figures S1-3 Supplemental
Table S1. Mapping statistics of RNA-Seq data from placenta and HBM2.0
tissues. Supplemental Table S2. Distribution of gene expression level
(FPKM) of RNA-Seq data from placenta and HBM2.0 tissues. Figure S1.
Distribution of gene expression values (FPKM) for all tissues examined in
the study. Figure S2. qRT-PCR validation of placenta-enriched SFs ESRP1
and MBNL3. Figure S3. Functional interaction network analysis of genes
with enriched expression (EE) and differential splicing (DS) that intersect
all three placental tissues: module 2. Circular node: a query gene.
Diamond-shaped node: a linker gene. Node color was determined based
on whether the query gene shows EE (green), DS (pink), or both (red).
The most significantly enriched pathways were highlighted in bigger
node size: integrin signaling pathway and ECM-receptor interaction
pathway.
Additional file 2: Table S3 Enriched pathways (FDR < 0.05 ) in the
whole network and submodules (module size > 50) from functional
interaction network analysis.
Additional file 3: Table S4 FPKM expression levels in all tissues for
the novel TARs identified from the placental tissues.
Additional file 4: Table S5 Exon inclusion levels and primer
sequences for exons selected for RT-PCR validation.
Additional file 5: Figure S4 RT-PCR analysis of $\mathbf{3 4}$ exons that
showed significant differential splicing (> 10\% difference in exon
inclusion level, FDR < $\mathbf{0 . 1}$ ) between placental and HBM2.0 tissues.
Figure S5. RT-PCR analysis of $\mathbf{2 1}$ ESRP1target exons.

\section{Abbreviations}

AS: Alternative splicing; DS: Differential splicing; EE: Enriched expression; Fl: Functional interaction; FPKM: Fragments Per Kilobase of gene per Million mapped fragments; GO: Gene Ontology; HBM2.0: Human Body Map 2.0; IUGR: Intrauterine growth restriction; MGI: Mouse Genome Informatics; PE: Preeclampsia; PTB: Preterm birth; TAR: Transcriptional active region.

\section{Acknowledgements}

We thank Elizabeth Kenkel for assistance with the PCR experiments and Roger Williamson for providing helpful comments on the manuscript. The HBM2.0 RNA-Seq data were kindly provided by the Gene Expression Applications research group at Illumina. The work was supported by 
Burroughs Wellcome Fund 1008841.01 (to JCM and YX), NIH grant HD52953 and HD57192 and grants from the March of Dimes (to JCM \#6-FY08-260 and \#21-FY06575; and to YX \#5-FY10-60).

We thank Elizabeth Kenkel for assistance with the PCR experiments and Roger Williamson for providing helpful comments on the manuscript. The HBM2.0 RNA-Seq data were kindly provided by the Gene Expression Applications research group at Illumina. The work was supported by Burroughs Wellcome Fund 1008841.01 (to JCM and YX), NIH grant HD52953 and HD57192 and grants from the March of Dimes (to JCM \#6-FY08-260 and \#21-FY06575; and to YX \#5-FY10-60).

\section{Author details}

'Department of Anatomy and Cell Biology, University of lowa, lowa City, IA 52242, USA. ${ }^{2}$ Department of Internal Medicine, University of lowa, lowa City, IA 52242, USA. ${ }^{3}$ Department of Pediatrics, University of lowa, lowa City, IA 52242, USA. ${ }^{4}$ Department of Biology, University of lowa, lowa City, IA 52242 USA. ${ }^{5}$ Department of Epidemiology, University of lowa, lowa City, IA 52242, USA. ${ }^{6}$ Department of Biomedical Engineering, University of lowa, lowa City, IA 52242, USA. ${ }^{7}$ Department of Biostatistics, University of lowa, lowa City, IA 52242, USA.

\section{Authors' contributions}

JK, KZ, JCM and YX designed the study. KZ, PJ, and JW analyzed data. JK isolated the tissues, prepared RNA for library preparation and sequencing. ZL conducted the RT-PCR validation experiments. JK, KZ, JCM and YX wrote the manuscript. All authors read and approved the final manuscript.

\section{Competing interests}

The authors declare that they have no competing interests.

Received: 2 December 2011 Accepted: 27 March 2012

Published: 27 March 2012

\section{References}

1. Kay HH, Nelson DM, Wang Y: The placenta: from development to disease. Chichester, West Sussex: Wiley-Blackwell; 2011.

2. Roberts DJ, Post MD: The placenta in pre-eclampsia and intrauterine growth restriction. J Clin Pathol 2008, 61(12):1254-1260.

3. Faye-Petersen OM: The placenta in preterm birth. Journal of Clinical Pathology 2008, 61(12):1261-1275.

4. Goldenberg RL, Culhane JF, lams JD, Romero R: Epidemiology and causes of preterm birth. Lancet 2008, 371(9606):75-84.

5. Martin JA, Hamilton BE, Ventura SJ, Osterman MJ, Kirmeyer S, Mathews TJ, Wilson E: Births: final data for 2009. Natl Vital Stat Rep 2011, 60(1):1-104.

6. Mikheev AM, Nabekura T, Kaddoumi A, Bammler TK, Govindarajan R, Hebert MF, Unadkat JD: Profiling gene expression in human placentae of different gestational ages: an OPRU Network and UW SCOR Study. Reprod Sci 2008, 15(9):866-877.

7. Haddad R, Tromp G, Kuivaniemi H, Chaiworapongsa T, Kim YM, Mazor M, Romero R: Human spontaneous labor without histologic chorioamnionitis is characterized by an acute inflammation gene expression signature. American journal of obstetrics and gynecology 2006, 195(2):394-405.

8. Sitras V, Paulssen RH, Gronaas H, Vartun A, Acharya G: Gene expression profile in labouring and non-labouring human placenta near term. Molecular Human Reproduction 2008, 14(1):61-65.

9. Sitras V, Paulssen RH, Gronaas H, Leirvik J, Hanssen TA, Vartun A, Acharya G: Differential Placental Gene Expression in Severe Preeclampsia. Placenta 2009, 30(5):424-433.

10. Sood R, Zehnder JL, Druzin ML, Brown PO: Gene expression patterns in human placenta. Proc Natl Acad Sci USA 2006, 103(14):5478-5483.

11. Knox K, Baker JC: Genomic evolution of the placenta using co-option and duplication and divergence. Genome Res 2008, 18(5):695-705.

12. Pan Q, Shai O, Lee LJ, Frey BJ, Blencowe BJ: Deep surveying of alternative splicing complexity in the human transcriptome by high-throughput sequencing. Nat Genet 2008, 40(12):1413-1415.

13. Wang ET, Sandberg R, Luo S, Khrebtukova I, Zhang L, Mayr C, Kingsmore SF, Schroth GP, Burge CB: Alternative isoform regulation in human tissue transcriptomes. Nature 2008, 456(7221):470-476.
14. Chen M, Manley JL: Mechanisms of alternative splicing regulation: insights from molecular and genomics approaches. Nature Reviews Molecular Cell Biology 2009, 10(11):741.

15. Wang GS, Cooper TA: Splicing in disease: disruption of the splicing code and the decoding machinery. Nat Rev Genet 2007, 8(10):749-761.

16. Caceres JF, Kornblihtt AR: Alternative splicing: multiple control mechanisms and involvement in human disease. Trends Genet 2002, 18(4):186-193.

17. Soleymanlou N, Wu Y, Wang JX, Todros T, letta F, Jurisicova A, Post M, Caniggia I: A novel Mtd splice isoform is responsible for trophoblast cell death in pre-eclampsia. Cell Death Differ 2005, 12(5):441-452.

18. Sela S, Itin A, Natanson-Yaron S, Greenfield C, Goldman-Wohl D, Yagel S, Keshet $E$ : A novel human-specific soluble vascular endothelial growth factor receptor 1: cell-type-specific splicing and implications to vascular endothelial growth factor homeostasis and preeclampsia. Circ Res 2008, 102(12):1566-1574

19. Heydarian M, McCaffrey T, Florea L, Yang Z, Ross MM, Zhou W, Maynard SE: Novel splice variants of sFlt1 are upregulated in preeclampsia. Placenta 2009, 30(3):250-255.

20. Wang Z, Gerstein M, Snyder M: RNA-Seq: a revolutionary tool for transcriptomics. Nat Rev Genet 2009, 10(1):57-63.

21. Trapnell C, Williams BA, Pertea G, Mortazavi A, Kwan G, van Baren MJ, Salzberg SL, Wold BJ, Pachter L: Transcript assembly and quantification by RNA-Seq reveals unannotated transcripts and isoform switching during cell differentiation. Nature Biotechnology 2010, 28(5):511-515.

22. Mortazavi A, Williams BA, McCue K, Schaeffer L, Wold B: Mapping and quantifying mammalian transcriptomes by RNA-Seq. Nat Methods 2008, 5(7):621-628

23. Su Al, Wiltshire T, Batalov S, Lapp H, Ching KA, Block D, Zhang J, Soden R, Hayakawa M, Kreiman $G$, et al: A gene atlas of the mouse and human protein-encoding transcriptomes. Proc Natl Acad Sci USA 2004, 101(16):6062-6067.

24. Blake JA, Bult CJ, Kadin JA, Richardson JE, Eppig JT: The Mouse Genome Database (MGD): premier model organism resource for mammalian genomics and genetics. Nucleic Acids Res 2011, , 39 Database: D842-848.

25. The Mouse Genome Informatics database. [http://www.informatics.jax.org/ ].

26. Dolan SM, Hollegaard MV, Merialdi M, Betran AP, Allen T, Abelow C, Nace J, Lin BK, Khoury MJ, loannidis JP, et al: Synopsis of preterm birth genetic association studies: the preterm birth genetics knowledge base (PTBGene). Public Health Genomics 2010, 13(7-8):514-523.

27. The Preterm Birth Genetics Knowledge Base. [http://bioinformatics.aecom. yu.edu/ptbgene/index.html].

28. Dennis G Jr, Sherman BT, Hosack DA, Yang J, Gao W, Lane HC, Lempicki RA: DAVID: Database for Annotation, Visualization, and Integrated Discovery. Genome Biol 2003, 4(5):P3.

29. The Database for Annotation, Visualization and Integrated Discovery. [http://david.abcc.ncifcrf.gov/].

30. Benirschke K: Pathology of the human placenta New York: Springer; 2012

31. Thomas PD, Campbell MJ, Kejariwal A, Mi H, Karlak B, Daverman R, Diemer K, Muruganujan A, Narechania A: PANTHER: a library of protein families and subfamilies indexed by function. Genome Research 2003, 13(9):2129-2141.

32. Thomas PD, Kejariwal A, Guo N, Mi H, Campbell MJ, Muruganujan A Lazareva-Ulitsky B: Applications for protein sequence-function evolution data: $\mathrm{mRNA} /$ protein expression analysis and coding SNP scoring tools. Nucleic Acids Research 2006, , 34 Web Server: W645-650.

33. Lash GE, Warren AY, Underwood S, Baker PN: Vascular endothelial growth factor is a chemoattractant for trophoblast cells. Placenta 2003 24(5):549-556

34. Baker PN, Krasnow J, Roberts JM, Yeo KT: Elevated serum levels of vascular endothelial growth factor in patients with preeclampsia. Obstet Gynecol 1995, 86(5):815-821.

35. Akercan F, Cirpan T, Terek MC, Ozcakir HT, Giray G, Sagol S, Karadadas N: The immunohistochemical evaluation of VEGF in placenta biopsies of pregnancies complicated by preeclampsia. Arch Gynecol Obstet 2008, 277(2):109-114.

36. Cirpan T, Akercan F, Terek MC, Kazandi M, Ozcakir HT, Giray G, Sagol S Evaluation of VEGF in placental bed biopsies from preeclamptic women by immunohistochemistry. Clin Exp Obstet Gynecol 2007, 34(4):228-231. 
37. Farina A, Sekizawa A, De Sanctis P, Purwosunu Y, Okai T, Cha DH, Kang JH, Vicenzi C, Tempesta A, Wibowo N, et al: Gene expression in chorionic villous samples at 11 weeks' gestation from women destined to develop preeclampsia. Prenat Diagn 2008, 28(10):956-961.

38. Farina A, Zucchini C, Sekizawa A, Purwosunu Y, de Sanctis P, Santarsiero G, Rizzo N, Morano D, Okai T: Performance of messenger RNAs circulating in maternal blood in the prediction of preeclampsia at 10-14 weeks. American journal of obstetrics and gynecology 2010, 203(6):575, e571-577.

39. Semczuk-Sikora A, Krzyzanowski A, Kwiatek M, Semczuk M: [Maternal serum concentration of placental growth factor (PIGF) and endothelial growth factor (VEGF) in pregnancies complicated by preeclampsia]. Ginekol Pol 2007, 78(11):873-876

40. Sandman CA, Glynn L, Schetter CD, Wadhwa P, Garite T, Chicz-DeMet A, Hobel C: Elevated maternal cortisol early in pregnancy predicts third trimester levels of placental corticotropin releasing hormone $(\mathrm{CRH})$ : priming the placental clock. Peptides 2006, 27(6):1457-1463.

41. Vitoratos N, Papadias K, Makrakis E, Christodoulakos G, Panoulis K, Creatsas G: Association between serum tumor necrosis factor-alpha and corticotrophin-releasing hormone levels in women with preterm labor. $J$ Obstet Gynaecol Res 2006, 32(5):497-501.

42. Grosso AR, Gomes AQ, Barbosa-Morais NL, Caldeira S, Thorne NP, Grech G, von Lindern M, Carmo-Fonseca M: Tissue-specific splicing factor gene expression signatures. Nucleic Acids Res 2008, 36(15):4823-4832.

43. Yeo GW, Coufal NG, Liang TY, Peng GE, Fu X-D, Gage FH: An RNA code for the FOX2 splicing regulator revealed by mapping RNA-protein interactions in stem cells. Nat Struct Mol Biol 2009, 16(2):130

44. Warzecha CC, Sato TK, Nabet B, Hogenesch JB, Carstens RP: ESRP1 and ESRP2 are epithelial cell-type-specific regulators of FGFR2 splicing. Mol Cell 2009, 33(5):591-601.

45. de la Grange P, Gratadou L, Delord M, Dutertre M, Auboeuf D: Splicing factor and exon profiling across human tissues. Nucleic Acids Res 2010, 38(9):2825-2838

46. Warzecha CC, Jiang P, Amirikian K, Dittmar KA, Lu H, Shen S, Guo W, Xing Y, Carstens RP: An ESRP-regulated splicing programme is abrogated during the epithelial-mesenchymal transition. EMBO J 2010, 29(19):3286-3300.

47. Bland CS, Wang ET, Vu A, David MP, Castle JC, Johnson JM, Burge CB, Cooper TA: Global regulation of alternative splicing during myogenic differentiation. Nucleic Acids Res 2010, 38(21):7651-7664.

48. Shen S, Won Park J, Huang J, Dittmar KA, Lu ZX, Zhou Q, Carstens RP, Xing Y: MATS: a Bayesian framework for flexible detection of differential alternative splicing from RNA-Seq data. Nucleic Acids Res 2012, doi:10.1093/nar/gkr1291.

49. Tamura RN, Cooper HM, Collo G, Quaranta V: Cell type-specific integrin variants with alternative alpha chain cytoplasmic domains. Proc Natl Acad Sci USA 1991, 88(22):10183-10187.

50. Lee EC, Lotz MM, Steele GD Jr, Mercurio AM: The integrin alpha 6 beta 4 is a laminin receptor. J Cell Biol 1992, 117(3):671-678.

51. Heinemann T, Bulwin GC, Randall J, Schnieders B, Sandhoff K, Volk HD, Milford E, Gullans SR, Utku N: Genomic organization of the gene coding for TIRC7, a novel membrane protein essential for T cell activation. Genomics 1999, 57(3):398-406.

52. Smirnova AS, Morgun A, Shulzhenko N, Silva ID, Gerbase-DeLima M: Identification of new alternative splice events in the TCIRG1 gene in different human tissues. Biochem Biophys Res Commun 2005, 330(3):943-949.

53. Hu Y, Leo C, Yu S, Huang BC, Wang H, Shen M, Luo Y, Daniel-Issakani S, Payan DG, Xu X: Identification and functional characterization of a novel human misshapen/Nck interacting kinase-related kinase, hMINK beta. The Journal of biological chemistry 2004, 279(52):54387-54397.

54. Wu G, Feng $X$, Stein $L$ : A human functional protein interaction network and its application to cancer data analysis. Genome Biology 2010, 11(5): R53-R53.

55. Girvan M, Newman ME: Community structure in social and biological networks. Proc Natl Acad Sci USA 2002, 99(12):7821-7826.

56. Guttman M, Garber M, Levin JZ, Donaghey J, Robinson J, Adiconis X, Fan L, Koziol MJ, Gnirke A, Nusbaum C, et al: Ab initio reconstruction of cell type-specific transcriptomes in mouse reveals the conserved multiexonic structure of lincRNAs. Nat Biotechnol 2010, 28(5):503-510.

57. Salomonis N, Nelson B, Vranizan K, Pico AR, Hanspers K, Kuchinsky A, Ta L, Mercola $M$, Conklin BR: Alternative splicing in the differentiation of human embryonic stem cells into cardiac precursors. PLoS Comput Biol 2009, 5(11):e1000553.

58. Yeo GW, Xu X, Liang TY, Muotri AR, Carson CT, Coufal NG, Gage FH: Alternative splicing events identified in human embryonic stem cells and neural progenitors. PLOS Comput Biol 2007, 3(10):1951-1967.

59. Delorme MA, Burrows RF, Ofosu FA, Andrew M: Thrombin regulation in mother and fetus during pregnancy. Semin Thromb Hemost 1992, 18(1):81-90.

60. Jabbour HN, Critchley HO: Potential roles of decidual prolactin in early pregnancy. Reproduction 2001, 121(2):197-205.

61. Randhawa RS: The insulin-like growth factor system and fetal growth restrictionn. Pediatr Endocrinol Rev 2008, 6(2):235-240.

62. Coan PM, Fowden AL, Constancia M, Ferguson-Smith AC, Burton GJ, Sibley CP: Disproportional effects of lgf2 knockout on placental morphology and diffusional exchange characteristics in the mouse. $J$ Physiol 2008, 586(Pt 20):5023-5032.

63. Prager S, Wollmann HA, Mergenthaler S, Mavany M, Eggermann K, Ranke MB, Eggermann T: Characterization of genomic variants in $\mathrm{CSH}$ and $\mathrm{GH} 2$, two candidate genes for Silver-Russell syndrome in 17q24q25. Genet Test 2003, 7(3):259-263.

64. Rull K, Laan M: Expression of beta-subunit of HCG genes during normal and failed pregnancy. Hum Reprod 2005, 20(12):3360-3368.

65. Henderson DJ, Bennett PR, Moore GE: Expression of human chorionic gonadotrophin alpha and beta subunits is depressed in trophoblast from pregnancies with early embryonic failure. Hum Reprod 1992, 7(10):1474-1478

66. Hunt JS, Langat DK, Mclntire RH, Morales PJ: The role of HLA-G in human pregnancy. Reprod Biol Endocrinol 2006, , 4 Suppl 1: S10.

67. Hunt JS, Petroff MG, McIntire RH, Ober C: HLA-G and immune tolerance in pregnancy. FASEB J 2005, 19(7):681-693.

68. Izumi-Yoneda N, Toda A, Okabe M, Koike C, Takashima S, Yoshida T, Konishi I, Saito S, Nikaido T: Alpha 1 antitrypsin activity is decreased in human amnion in premature rupture of the fetal membranes. Mol Hum Reprod 2009, 15(1):49-57.

69. Niknejad H, Peirovi H, Jorjani M, Ahmadiani A, Ghanavi J, Seifalian AM: Properties of the amniotic membrane for potential use in tissue engineering. Eur Cell Mater 2008, 15:88-99.

70. Burrows TD, King A, Loke YW: Trophoblast migration during human placental implantation. Hum Reprod Update 1996, 2(4):307-321.

71. Massague J: How cells read TGF-beta signals. Nat Rev Mol Cell Biol 2000, 1(3):169-178.

72. Fryer BH, Simon MC: Hypoxia, HIF and the placenta. Cell Cycle 2006, 5(5):495-498

73. Poschl E, Schlotzer-Schrehardt U, Brachvogel B, Saito K, Ninomiya Y, Mayer U: Collagen IV is essential for basement membrane stability but dispensable for initiation of its assembly during early development. Development 2004, 131(7):1619-1628.

74. Harkness ML, Harkness RD: The growth of collagen in the foetus, placenta and foetal membranes of the rat. J Physiol 1955, 128(2):225-234

75. Shen S, Lin L, Cai JJ, Jiang P, Kenkel EJ, Stroik MR, Sato S, Davidson BL, Xing Y: Widespread establishment and regulatory impact of Alu exons in human genes. Proc Natl Acad Sci USA 2011, 108(7):2837-2842.

76. Langmead B, Trapnell C, Pop M, Salzberg SL: Ultrafast and memoryefficient alignment of short DNA sequences to the human genome. Genome Biol 2009, 10(3):R25.

77. Huang DW, Sherman BT, Lempicki RA: Systematic and integrative analysis of large gene lists using DAVID bioinformatics resources. Nat Protoc 2009, 4(1):44-57

78. Lu ZX, Jiang $P$, Cai JJ, Xing $Y$ : Context-dependent robustness to $5^{\prime}$ splice site polymorphisms in human populations. Hum Mol Genet 2011, 20(6):1084-1096.

79. Schuelke M: An economic method for the fluorescent labeling of PCR fragments. Nat Biotechnol 2000, 18(2):233-234.

80. Livak KJ, Schmittgen TD: Analysis of relative gene expression data using real-time quantitative PCR and the 2(-Delta Delta $C(T))$ Method. Methods 2001, 25(4):402-408.

81. Smoot ME, Ono K, Ruscheinski J, Wang P-L, Ideker T: Cytoscape 2.8: new features for data integration and network visualization. Bioinformatics 2010, 27(3):431-432. 
82. Yoon J, Blumer A, Lee K: An algorithm for modularity analysis of directed and weighted biological networks based on edge-betweenness centrality. Bioinformatics 2006, 22(24):3106-3108.

doi:10.1186/1471-2164-13-115

Cite this article as: Kim et al:: Transcriptome landscape of the human placenta. BMC Genomics 2012 13:115.

Submit your next manuscript to BioMed Central and take full advantage of:

- Convenient online submission

- Thorough peer review

- No space constraints or color figure charges

- Immediate publication on acceptance

- Inclusion in PubMed, CAS, Scopus and Google Scholar

- Research which is freely available for redistribution

Submit your manuscript at www.biomedcentral.com/submit 